\title{
Coordination of Locomotion with Voluntary Movements in Humans
}

\author{
Yuri P. Ivanenko, ${ }^{1}$ Germana Cappellini, ${ }^{1}$ Nadia Dominici, ${ }^{1,2}$ Richard E. Poppele, ${ }^{4}$ and Francesco Lacquaniti ${ }^{1,2,3}$ \\ ${ }^{1}$ Department of Neuromotor Physiology, Scientific Institute Foundation Santa Lucia, 00179 Rome, Italy, ${ }^{2}$ Department of Neuroscience and ${ }^{3}$ Centre of Space \\ Bio-medicine, University of Rome Tor Vergata, 00173 Rome, Italy, and ${ }^{4}$ Department of Neuroscience, University of Minnesota, Minneapolis, Minnesota \\ 55455
}

\begin{abstract}
Muscle activity occurring during human locomotion can be accounted for by five basic temporal activation patterns in a variety of locomotion conditions. Here, we examined how these activation patterns interact with muscle activity required for a voluntary movement. Subjects produced a voluntary movement during locomotion, and we examined the resulting kinematics, kinetics, and EMG activity in 16-31 ipsilateral limb and trunk muscles during the tasks. There were four voluntary tasks added to overground walking $(\sim 5$ $\mathrm{km} / \mathrm{h}$ ) in which subjects kicked a ball, stepped over an obstacle, or reached down and grasped an object on the floor (weight support on either the right or the left foot). Statistical analyses of EMG waveforms showed that the five basic locomotion patterns were invariantly present in each task, although they could be differently weighted across muscles, suggesting a characteristic locomotion timing of muscle activations. We also observed a separate activation that was timed to the voluntary task. The coordination of locomotion with the voluntary task was accomplished by combining activation timings that were associated separately with the voluntary task and locomotion. Activation associated with the voluntary tasks was either synchronous with the timing for locomotion or had additional activations not represented in the basic locomotion timing. We propose that this superposition of an invariant locomotion timing pattern with a voluntary activation timing may be consistent with the proposal suggesting that compound movements are produced through a superposition of motor programs.
\end{abstract}

Key words: EMG activity; motor primitives; central pattern generators; spinal cord; human locomotion; walking

\section{Introduction}

The question of how muscle activity is coordinated to produce directed movements is central to an understanding of motor control. It is generally accepted that the nervous system adopts strategies that reduce the complexity of this problem (Bernstein, 1967; Lacquaniti et al., 1999, 2002; Latash, 1999; Orlovsky et al., 1999; Giszter et al., 2001; Hultborn, 2001; Tresch et al., 2002). One recent line of investigation has proposed that this is achieved by using a few motor primitives that may be combined in various ways to produce movements in the frog, rat, and cat (Bizzi et al., 2000; Kargo and Giszter, 2000; d'Avella et al., 2003; Hart and Giszter, 2004; Lemay and Grill, 2004). A similar concept of a limited basic set of muscle synergies was introduced also for balance control in the cat and in humans (Krishnamoorthy et al., 2003; Ting and Macpherson, 2005).

Mammalian locomotion is another behavior that might provide insights about motor control strategies (Shiavi and Griffin, 1981; Patla, 1985; Patla et al., 1985; Wootten et al., 1990; Davis

Received Jan. 26, 2005; revised June 16, 2005; accepted June 17, 2005.

This work was supported by the Italian Ministry of Health, the Italian Ministry of University and Research, and the Italian Space Agency. We thank Dr. G. Bosco for comments on a previous version of this manuscript, Dr. A. d'Avella for helpful discussion of the statistical methods, and S. Gravano for help with some of the experiments.

Correspondence should be addressed to Dr. Yuri P. Ivanenko, Department of Neuromotor Physiology, Scientific Institute Foundation Santa Lucia, 306 via Ardeatina, 00179 Rome, Italy. E-mail: y.ivanenko@hsantalucia.it. DOI:10.1523/JNEUROSCI.1327-05.2005

Copyright $\odot 2005$ Society for Neuroscience $\quad 0270-6474 / 05 / 257238-16 \$ 15.00 / 0$ and Vaughan, 1993; Lacquaniti et al., 2002; Yakovenko et al., 2002; Poppele and Bosco, 2003). Consistent with some of the primitives ideas, we and others (Davis and Vaughan, 1993; Olree and Vaughan, 1995; Ivanenko et al., 2003, 2004) have shown that muscle activity during human locomotion is driven by a few (approximately five) temporal activation components. Each activation component describes a short period of synchronous activation or relaxation of a particular set of muscles, and it represents a characteristic timing rather than a characteristic muscle synergy associated with each movement component. We proposed that this sequence of activation components is specifically associated with locomotion movements, because it was unaltered by changes in locomotion speed or by body weight support during locomotion.

Here, we tested this idea further by having subjects produce a voluntary movement during locomotion. We designed two sets of voluntary motor tasks to be performed during normal overground locomotion. One set involved voluntary perturbations of normal gait, stepping over an obstacle, and kicking a ball, thereby modifying the footpath or requiring extra force. The other set of tasks involved coordinated whole-body movements to reach down and grasp an object on the floor while walking. To examine muscle activation, we recorded EMG activity from leg and body muscles and applied a factor or component analysis to determine elements of activation timing.

We can anticipate three possibilities for muscle activation in 
these tasks. One is that the five activation components for locomotion will account for the muscle activity in all tasks. This could be expected if the neural control were tightly coupled to the rhythmic pendulum-like behavior of the biomechanical system, so that muscles were activated only in certain phases of the locomotion cycle and in response to proprioceptive feedback (Prochazka et al., 2002). Although this seems a likely outcome for the first two tasks, it seems less likely for the stoop-and-grasp tasks. For those we might anticipate different or reconfigured activation profiles because muscle forces and joint torques as well as proprioceptive feedback can all be quite different. This result would imply that the voluntary command and/or the proprioceptive feedback have a direct influence on the timing of the activation components. A third possibility is that the two aspects of the task, namely the locomotion and the voluntary movement, would each have their own temporal activation components that are superimposed in the combined task, as envisioned by the motor primitives proposal. This result could be expected if the characteristic activation timing for locomotion were generated separately from any voluntary activation timing, although a coupling of corticospinal with cervical and thoraco-lumbar propriospinal circuits (Dietz, 2002; Zehr and Duysens, 2004) may result in partial synchronization of activation components. Additional temporal components would imply voluntary activation timings that are not represented in the locomotion sequence.

\section{Materials and Methods Subjects}

Eight healthy subjects [five males and three females, 28-44 years old, $58 \pm 11 \mathrm{~kg}$ (mean $\pm \mathrm{SD}), 1.69 \pm 0.06 \mathrm{~m}]$ volunteered for the experiments. All subjects were right-leg dominant. The studies conformed to the Declaration of Helsinki, and informed consent was obtained from all participants according to the protocol of the Ethics Committee of the Santa Lucia Institute.

\section{Walking conditions}

Five walking conditions were recorded (see Fig. 1, top). Presentation was randomized across subjects. In all conditions, subjects stepped on a force plate located in the middle of a $7 \mathrm{~m}$ walkway. All voluntary tasks were executed during a single step cycle that began with right-heel strike on the force plate. Before recordings, subjects practiced for a few minutes in walking at approximately the same speed in all conditions. An appropriate initial position was chosen so that subjects stepped on the force plate with the right foot. Subjects were asked to continue to walk when performing a voluntary task. In each condition, five trials were recorded.

\section{Normal walking}

Subjects were asked to walk at a natural speed along a walkway while looking forward. Subjects usually performed several steps with approximately a steady-state velocity (on average, $5.2 \pm 0.8 \mathrm{~km} \mathrm{~h}^{-1}$ ) in the middle of the walkway, where they stepped on the force plate.

\section{Kicking a ball}

Subjects were asked to walk at a natural speed and kick with the right leg a stationary ball placed in the locomotion path ("kick" condition). They were asked to strike the ball at a target located $\approx 4 \mathrm{~m}$ in front of them (an experimenter who had to catch the ball). The ball (diameter, $30 \mathrm{~cm} ; 0.44$ $\mathrm{kg}$ ) was placed $\sim 50 \mathrm{~cm}$ ahead of the force plate so that we recorded the ground reaction forces under the right foot during stance just before the kick. Kick force was limited (to avoid harm to surroundings) but sufficient to propel the ball in front of the subject. The force of the kick was, on average, $81 \pm 16 \mathrm{~N}$ (estimated as $m \times a$, where $m$ is the mass of the ball and $a$ is the maximum acceleration of the midpoint between the two markers placed on the opposite sides of the ball), and the resulting initial ball velocity was $8.2 \pm 1.4 \mathrm{~m} \mathrm{~s}^{-1}$.

\section{Stepping over an obstacle}

Subjects were asked to walk at a natural speed and step over an obstacle with the right leg ("obstacle" condition). The obstacle $(30 \mathrm{~cm}$ height, made of foam rubber) was located at the end of the force plate so that we recorded the ground reaction forces under the right foot during stance before the foot lift.

Stooping with the right weight-bearing leg and grasping an object Subjects were asked to walk at a natural speed and grasp a small object located on the floor with the right hand while the right leg was the weight-bearing leg ("stoop-right" condition). The object (soft ball; diameter, $8 \mathrm{~cm}$ ) was located in the middle and on the right side of the force plate so that we could record the ground reaction forces under the right foot during grasping. Because the location of the object and the right foot placement during grasping were stereotyped across all trials (as a result of the appropriate choice of the initial position), the kinematic behavior during the task was similar across subjects.

Stooping with the left weight-bearing leg and grasping an object Subjects were asked to walk at a natural speed and grasp a small object located on the floor with the right hand while the left leg was the weightbearing leg ("stoop-left" condition). The object (soft ball; diameter, 8 $\mathrm{cm}$ ) was located immediately ahead the force plate (by $\sim 10 \mathrm{~cm}$ ) so that we could record the ground reaction forces under the right foot during stance just before grasping.

\section{Additional experiments}

Because voluntary tasks could evoke moderate changes in the walking speed (deceleration or acceleration with respect to the previous step), we did an additional experiment to determine whether the EMG patterns and underlying basic components would change substantially during gait acceleration or deceleration. We tested three subjects by recording EMGs while walking on a treadmill (EN-MILL 3446.527; Bonte Zwolle BV, Zwolle, The Netherlands) with different speed conditions. We used a constant speed at $5 \mathrm{~km} \mathrm{~h}^{-1}$ (constant-speed condition) and a computercontrolled speed program that linearly increased and then decreased the belt speed between 1 and $7 \mathrm{~km} \mathrm{~h}^{-1}$ (ramp-speed condition). We recorded three trials from each subject in each condition. The walking surface of the treadmill was $1.5 \mathrm{~m}$ long, $0.6 \mathrm{~m}$ wide, and $0.15 \mathrm{~m}$ above the ground. The subjects were instructed to follow the changes in speed by remaining in place with respect to the treadmill when the belt velocity was changing. Deceleration (and acceleration) was set to 0.4 or $0.7 \mathrm{~km}$ $\mathrm{h}^{-1}$ per second in separate trials. These changes in speed were compatible with those observed during voluntary tasks. In the ramp experiment, we calculated the mean belt speed during each gait cycle to analyze motor patterns at the same cycle speed of $5 \mathrm{~km} \mathrm{~h}^{-1}$ in all three conditions (constant-speed condition, acceleration ramp, and deceleration ramp).

In another set of experiments, we had subjects perform two of the voluntary components while standing, instead of during locomotion. For one of these, we asked three subjects to perform a stoop-right task from the standing position using the same cadence they used during locomotion. For this stoop-in-place task, the right foot was placed slightly forward by $30-40 \mathrm{~cm}$ to mimic the body configuration in the stoop-right task. For another experiment, we asked two subjects to perform a stepin-place task to produce the same voluntary movement (right foot lift) they used to clear the obstacle during locomotion, again at the same cadence. We then determined the EMG activation components for each experiment.

\section{Data recording}

Kinematic data were recorded bilaterally at $100 \mathrm{~Hz}$ by means of the Vicon-612 system (Vicon, Oxford, UK). The spatial accuracy of the system is better than $1 \mathrm{~mm}$ (root mean square). Nine television cameras were spaced around the walkway. Infrared reflective markers (diameter, $1.4 \mathrm{~cm}$ ) were attached on each side of the subject to the skin overlying the following landmarks: gleno-humeral joint $(\mathrm{GH})$, the midpoint between the anterior and the posterior superior iliac spine [ilium (IL)], greater trochanter (GT), lateral femur epicondyle (LE), lateral malleolus (LM), and fifth metatarso-phalangeal joint (VM). In addition, we attached two 
Table 1. Muscles recorded for each subject

\begin{tabular}{|c|c|c|c|c|c|c|c|c|}
\hline Muscle & $E D$ & FK & $\mathrm{GC}$ & MZ & SG & ND & $\mathrm{YI}$ & $\mathrm{BM}$ \\
\hline SPLE & $X$ & & & & $X$ & * & $X$ & $X$ \\
\hline TRAPS & $X$ & & & $X$ & $X$ & * & $X$ & $X$ \\
\hline TRAPI & $X$ & & & & & $X$ & $X$ & $X$ \\
\hline DELTA & $X$ & & & & $X$ & * & $X$ & $X$ \\
\hline DELTP & $X$ & & & & $X$ & * & $X$ & $X$ \\
\hline $\mathrm{BIC}$ & * & & & & $X$ & * & * & $X$ \\
\hline TRIC & $X$ & & & & $X$ & * & $X$ & $X$ \\
\hline LD & $X$ & $X$ & $X$ & $X$ & $X$ & $X$ & $X$ & $X$ \\
\hline EST1 & $X$ & & & & $X$ & $X$ & $X$ & $X$ \\
\hline EST9 & $X$ & & & & & $X$ & $X$ & $X$ \\
\hline ESL2 & $X$ & $X$ & $X$ & $X$ & $X$ & $X$ & $X$ & $X$ \\
\hline RAS & $X$ & * & * & & $X$ & * & $X$ & $X$ \\
\hline $\mathrm{OE}$ & * & $X$ & $X$ & $X$ & $X$ & $x$ & * & $X$ \\
\hline 이 & $X$ & & & & & * & $X$ & $X$ \\
\hline Gmed & $X$ & & & & $X$ & $X$ & $X$ & $X$ \\
\hline GM & $X$ & $X$ & $X$ & $X$ & $X$ & $x$ & $X$ & $X$ \\
\hline ILIO & $X$ & & & & $X$ & $X$ & $X$ & $X$ \\
\hline TFL & $X$ & $X$ & * & $X$ & $X$ & $X$ & $X$ & $X$ \\
\hline$B F$ & $X$ & $X$ & $X$ & $X$ & $X$ & $x$ & $X$ & $X$ \\
\hline ST & $X$ & $X$ & $X$ & $X$ & $X$ & $X$ & $X$ & $X$ \\
\hline ADD & $X$ & $X$ & $X$ & $X$ & $X$ & $X$ & * & $X$ \\
\hline SART & $X$ & * & * & $X$ & $X$ & $X$ & $X$ & $X$ \\
\hline Vmed & $x$ & & & & $X$ & & & \\
\hline Vlat & $X$ & $X$ & $X$ & $X$ & $X$ & $X$ & $X$ & $X$ \\
\hline $\mathrm{RF}$ & $X$ & $X$ & $X$ & $X$ & $X$ & $x$ & $X$ & $X$ \\
\hline TA & $X$ & $X$ & $X$ & $X$ & $X$ & $X$ & $X$ & $X$ \\
\hline PERL & $X$ & $X$ & $X$ & $X$ & $X$ & $X$ & $X$ & $X$ \\
\hline$M G$ & $X$ & & & & $X$ & & $X$ & \\
\hline LG & $X$ & $X$ & $X$ & $X$ & $X$ & $X$ & $X$ & $X$ \\
\hline Sol & $X$ & $X$ & $X$ & $X$ & $X$ & $X$ & $X$ & $X$ \\
\hline FDB & & & & & & $X$ & $X$ & $X$ \\
\hline
\end{tabular}

$X$, Mean EMG activity was $>2 \mu \mathrm{V}$ in all conditions; ${ }^{*}$, mean EMG activity was $<2 \mu \mathrm{V}$ (considered to be a noise) in some conditions and therefore was not included in the FA for those conditions.

markers to the wrist and elbow of the right arm, as well as to the ball (kick condition), obstacle (obstacle condition), and object (stoop condition).

The ground reaction forces $\left(F_{\mathrm{x}}, F_{\mathrm{y}}\right.$, and $\left.F_{\mathrm{z}}\right)$ under the right foot were recorded at $1000 \mathrm{~Hz}$ by a force platform $(0.9 \times 0.6 \mathrm{~m}$; Kistler 9287B; Kistler, Zurich, Switzerland).

Electromyographic activity was recorded by means of surface electrodes from 16-31 muscles simultaneously on the right side of the body in each of eight normal subjects. We recorded from slightly different sets of muscles in the eight subjects (Table 1). The following 15 muscles were recorded from all eight subjects: tibialis anterior (TA); gastrocnemius lateralis (LG); soleus (Sol); peroneus longus (PERL); vastus lateralis (Vlat); rectus femoris (RF); sartorius (SART); biceps femoris (long head) (BF); semitendinosus (ST); adductor longus (ADD); tensor fascia latae (TFL); gluteus maximus (GM); erector spinae, recorded at L2 (ESL2); external oblique (OE); and latissimus dorsi (LD). The following 16 muscles were recorded from two to five subjects (Table 1): flexor digitorum brevis (FDB); gastrocnemius medialis (MG); vastus medialis (Vmed); iliopsoas (ILIO); gluteus medius (Gmed); internal oblique (OI); rectus abdominis, superior portion (RAS); erector spinae, recorded at T1 and T9 (EST1 and EST9, respectively); biceps brachii (BIC); triceps brachii (TRIC); deltoideus, anterior and posterior portions (DELTA and DELTP, respectively); trapezius, inferior and superior portions (TRAPS and TRAPI, respectively); and splenius (SPLE). The activity was recorded using active electrodes (model DE2.1; Delsys, Boston, MA) applied to lightly abraded skin over the respective muscle belly. Electrode placement was as follows: for the ES muscle, $2 \mathrm{~cm}$ lateral to the spinous process; for Sol, $\sim 2 \mathrm{~cm}$ distal to the medial head of the gastrocnemius; for RAS, $\sim 3 \mathrm{~cm}$ lateral of the umbilicus (Winter, 1991). For many muscles, electrode placement was chosen carefully so as to minimize cross talk from adjacent muscles during isometric contractions. Before the electrodes were placed, the subject was instructed about how to selectively activate each muscle (Kendall et al., 1993), while EMG signals were mon- itored. The signals were amplified (10,000×), filtered (20-450 Hz; Bagnoli 16; Delsys), and sampled at $1000 \mathrm{~Hz}$. Sampling of kinematic, force platform, and EMG data were synchronized.

In addition, we recorded intramuscular EMGs in one subject to control the effectiveness of cross-talk rejection by our surface recordings during normal walking, obstacle, and stoop-right conditions. We used a single-needle technique (Basmajian and De Luca, 1985) to record activity within the following seven leg muscles: LG, TA, PERL, SART, BF, ST, and TFL. Twisted $50-\mu \mathrm{m}$-diameter wire pairs were threaded through a 27 gauge hypodermic needle that was used to insert the wires and subsequently withdrawn to leave the wires in place (Ivanenko et al., 2004). The wires were bent back to form hooks on the opposite sites of the shaft to prevent their direct contact and to provide a desirable orientation of the electrodes along the muscle fibers. Surface EMG electrodes were also placed within $2-3 \mathrm{~cm}$ of the intramuscular insertion points along the direction of muscle fibers.

At the end of the recording session, anthropometric measurements were taken on each subject. These included the mass and stature of the subject and the length and circumference of the main segments of the body (Zatsiorsky et al., 1990).

\section{Data analysis}

The gait cycle under consideration was always defined with respect to the right leg movement, beginning with right-heel strike. The body was modeled as an interconnected chain of rigid segments: IL-GT for the pelvis, GT-LE for the thigh, LE-LM for the shank, and LM-VM for the foot. The elevation angle of each segment in the sagittal plane corresponds to the angle between the projected segment and the vertical. The main limb axis was defined as GT-LM. The elevation angle of each segment (including the limb axis) corresponds to the angle between the segment projected on the sagittal plane and the vertical (positive in the forward direction, i.e., when the distal marker falls anterior to the proximal one). Gait cycle was defined as the time between two successive heel strikes of the right leg. The first heel strike was determined from the force plate recordings (when the vertical ground reaction force exceeded $7 \%$ of the body weight). The timing of the lift off was determined analogously. The end of the gait cycle (the end of the swing phase) was determined as the maximum elevation of the limb axis of the right leg. The time of maximum and minimum elevation of the limb axis generally corresponds to heelcontact and toe-off (stance to swing transition), respectively (Borghese et al., 1996). These kinematic criteria were verified by comparison with foot strike and lift-off events measured from the changes of the vertical force around a fixed threshold. In general, the difference between the time events measured from kinematics and kinetics was $<2 \%$. They were used to identify the "stance" and "swing" phases for the left leg during the same gait cycle (determined by the right leg).

The walking speed and its variation throughout the gait cycle (Table 2) were estimated by computing the instantaneous velocity of the center of body mass (COM). COM position was derived as follows:

$$
\mathrm{COM}=\frac{\sum_{i}^{n} m_{\mathrm{i}} \times \vec{r}_{\mathrm{i}}}{\sum_{i}^{n} m_{\mathrm{i}}}
$$

where $m_{\mathrm{i}}$ and $\vec{r}_{\mathrm{i}}$ are the mass and position of the center of mass of each body segment, respectively, derived using measured kinematics, anthropometric data taken on each subject (see above), and regression equations proposed by Zatsiorsky et al. (1990) for adults. Variations in speed throughout the gait cycle were computed as the amplitude of horizontal COM speed changes within the gait cycle. During normal walking, they result from an exchange between the potential and kinetic energies of the COM because of the inverted pendulum mechanism of walking. During voluntary tasks, they result also from decelerations and accelerations related to the performance of these additional voluntary tasks.

The timing of the voluntary task (Table 2 , last column) was estimated 
Table 2. General characteristics of stepping (mean \pm SD)

\begin{tabular}{llllll}
\hline Condition & $\begin{array}{l}\text { Mean walking } \\
\text { speed }\left(\mathrm{km} \mathrm{h}^{-1}\right)\end{array}$ & $\begin{array}{l}\text { Variations in speed over } \\
\text { the gait cycle }\left(\mathrm{km} \mathrm{h}^{-1}\right)\end{array}$ & $\begin{array}{l}\text { Cycle } \\
\text { duration }(\mathrm{s})\end{array}$ & $\begin{array}{l}\text { Stance duration } \\
\text { (\% cycle) }\end{array}$ & $\begin{array}{l}\text { Timing of voluntary } \\
\text { task (\% cycle) }\end{array}$ \\
\hline Normal walking & $5.2 \pm 0.8$ & $1.6 \pm 0.5$ & $1.04 \pm 0.13$ & $61 \pm 2 \%$ & \\
Kick & $5.9 \pm 0.3$ & $1.3 \pm 0.3$ & $1.00 \pm 0.07$ & $55 \pm 2 \%$ & $70 \pm 4 \%$ \\
Obstacle & $5.0 \pm 0.9$ & $1.4 \pm 0.5$ & $1.12 \pm 0.12$ & $53 \pm 3 \%$ & $56 \pm 4 \%$ \\
Stoop right & $5.2 \pm 0.9$ & $2.3 \pm 0.2$ & $1.15 \pm 0.12$ & $66 \pm 3 \%$ & $30 \pm 5 \%$ \\
Stoop left & $5.5 \pm 0.8$ & $2.0 \pm 0.1$ & $1.17 \pm 0.07$ & $64 \pm 2 \%$ & $57 \pm 7 \%$ \\
\hline
\end{tabular}

Cycle duration, stance duration, and timing of the voluntary task refer to the right-leg movement.

as the onset of the horizontal ball movement in the kick condition, the onset of the foot (VM marker) elevation in the obstacle task, and the onset of the vertical object movement in the stoop tasks.

The EMG records were rectified and low-pass filtered using a zero-lag Butterworth filter with a cutoff of $5 \mathrm{~Hz}$. Data were time-interpolated over individual gait cycles to fit a normalized 200-point time base.

\section{Intersegmental coordination}

The intersegmental coordination was evaluated in position space as described previously (Borghese et al., 1996; Bianchi et al., 1998). The temporal changes of the elevation angles at the thigh, shank, and foot covary during walking. When these angles are plotted one versus the others in a three-dimensional graph, they describe a path that can be fitted (in the least-square sense) by a plane over each gait cycle. To this end, we computed the covariance matrix of the ensemble of time-varying elevation angles (after subtraction of their mean value) over each gait cycle. The three eigenvectors $\mathrm{u}_{1}-\mathrm{u}_{3}$, rank-ordered on the basis of the corresponding eigenvalues, correspond to the orthogonal directions of maximum variance in the sample scatter. The first two eigenvectors, $\mathrm{u}_{1}$ and $\mathrm{u}_{2}$, lie on the best-fitting plane of angular covariation. The third eigenvector $\left(u_{3}\right)$ is the normal to the plane and defines the plane orientation. For each eigenvector, the parameters $u_{\mathrm{it}}, u_{\mathrm{is}}$, and $u_{\mathrm{if}}$ correspond to the direction cosines with the positive semi-axis of the thigh, shank, and foot angular coordinates, respectively. The orientation of the covariation plane in each condition was compared both across all subjects and with the mean orientation of the corresponding plane in the normal walking condition. In some conditions (stoop right and left), we evaluated the intersegmental coordination separately for the right and left legs and for the stance and swing phases because it differed significantly.

\section{Joint moments of force}

The moments of forces (with extensor moments being positive) at the ankle, knee, and hip joints of the right leg were calculated using measured kinematics, force plate data, anthropometric data taken on each subject, and the traditional Newton-Euler inverse dynamics model (Bresler and Frankel, 1950). The net support moment was computed as a sum of these three moments (Winter, 1991). The moments were normalized to the body mass.

\section{Factor analysis}

We applied a principal component analysis (PCA) to each of several data sets consisting of normalized EMG patterns over a step cycle. The factor analysis (FA) used here has been thoroughly described in our previous reports (Ivanenko et al., 2003, 2004). Briefly, the steps involve calculation of the correlation matrix, extraction of the initial principal components (PCs), application of the varimax rotation, calculation of factors scores (referred to as activation components here), factor loadings (weighting coefficients across muscles), and percentage of variance accounted for (VAF) by each factor in the total data set. The aim of the FA is to represent the original EMG data set E $(m \times t$ matrix, where $m$ is the number of muscles and $t=200$ for all conditions, because EMG data were timeinterpolated over individual gait cycles to fit a normalized 200-point time base) as a linear combination of $n$ basic temporal components $(n<m)$ :

$$
\mathrm{E}=W C+\text { residual, }
$$

where $W$ are weighting coefficients or loadings $(m \times n$ matrix $)$ and $C$ are basic components $(n \times t$ matrix).
The PCs were expressed using a varimax rotation to minimize the number of variables with high loadings on each component (Kaiser, 1974). This has the effect of simplifying the interpretation of the PCs because the waveforms of the rotated components are more similar to those of the EMGs than are the basic PC waveforms (Davis and Vaughan, 1993; Chau, 2001). In the FA, the basic waveforms are not specified in advance as in a Fourier series expansion. Instead, they are determined by the structure of the data waveforms.

Communalities, the Bartlett's test of sphericity, and the Kaiser-MeyerOlkin (KMO) measure were used to assess whether the data set is adequate for the FA. The communality (squared multiple correlation of an item with all other items) is the proportion of variance of a particular variable that is attributable to common factors shared with other variables and should be quite high. The Bartlett's test of sphericity tests the hypothesis that the correlation matrix comes from a population in which the variables are independent (i.e., an identity matrix). Rejection of the independence hypothesis is an indication that the data are adequate to the FA. The KMO measure compares the magnitudes of the observed correlation coefficients to the magnitudes of the partial correlation coefficients. This indicator should be $\geq 0.5$ (Kaiser, 1974; Merkle et al., 1998). In all of our conditions, the Bartlett's test of sphericity $(p<0.001)$, the $\mathrm{KMO}$ measure (ranging from 0.55 to 0.75 ), and the communalities $(>0.997)$ indicated that a FA was appropriate in all cases.

In some conditions, not all muscles were active in all subjects (Table 1). To avoid contribution of noisy EMGs on the shape of the basic components (because the FA uses a correlation of muscle activation patterns), we excluded the EMGs with the mean values $<2 \mu \mathrm{V}$ (considered to be a noise). Eigenvectors of the correlation matrix with the corresponding eigenvalues less than unity are usually considered to describe noise (Davis and Vaughan, 1993). This criterion was proposed by Kaiser (1974) and is probably the one most widely used. In practice, an additional important aspect is the extent to which a solution is interpretable. Therefore, one usually examines several solutions with more or fewer components and chooses the one that makes the best "sense." In the present study, unless a component explained at least as much as $3 \%$ of the total variance, we dropped it. The higher-order components were generally variable and not significant (see Results).

To assess similarities of activation components across subjects and conditions, we used the following parameters: correlation coefficient of the time courses of the activation component in different conditions, timing of the main peak, and the width of the main peak. The five basic components in the normal walk condition were ordered according to the timing of the main peak. The first five components in other conditions were also ordered by their best matching to the five averaged components obtained in the normal walk, whereas additional components were plotted separately. The timing of the main peak was expressed in the percentage cycle. The width of the main peak was estimated by measuring the full-width at half-maximum (FWHM). The half of maximum was calculated as a point corresponding to the mid-height between the peak value and the mean value between two minima (one to the left, another one to the right with respect to the main peak). In the case of "boundary" peaks (close to the heel strike), we used only the descending or ascending part of the component profile to calculate the half-width at half-maximum and then multiplied it by 2, under the assumption of the symmetrical profile.

Data filtering ( $5 \mathrm{~Hz}$ low-pass) could influence the VAF by a particular component, but it does not seem to affect the overall shape of the temporal components (Ivanenko et al., 2004).

A FA could theoretically be compromised by electrical cross talk among adjacent muscle recordings. However, if cross talk did exist, it would most likely have affected only the weighting coefficients assigned to each component in accounting for the activity of a muscle that was contaminated by cross talk. In the previous study (Ivanenko et al., 2004), we recorded simultaneously surface and intramuscular EMGs to control the effectiveness of cross-talk rejection by our surface recordings and a possible effect of cross talk on the PCs. The recording site (intramuscular 
or surface) had no significant effect on the waveform of the basic temporal components.

\section{Independent component analysis and non-negative matrix factorization}

Different statistical methods have been developed to assess a linear decomposition of the original set of data based on different assumptions. Thus, the FA with varimax rotation constrains the analysis to orthogonal (uncorrelated) factors. Although the FA of EMG activity was thoroughly documented for human locomotion and we could thus compare our results with those reported in the literature (Davis and Vaughan, 1993; Olree and Vaughan, 1995; Merkle et al., 1998; Ivanenko et al., 2003, 2004), we also performed two other decomposition-related statistical analyses: independent component analysis (ICA) and non-negative matrix factorization (NMF). All methods were performed task by task, and the results across tasks were compared.

We performed an informax ICA (Bell and Sejnowski, 1995) using the function "runica" in the EEGLAB package (version 4.5; www.sccn.ucsd. edu/eeglab/) (Delorme and Makeig, 2004) running on Matlab version 7. The rationale for this analysis is that unlike PCA (FA), ICA aims at extracting unknown hidden components from multivariate data using only the assumption that the unknown components are mutually independent.

We also applied an NMF using the matrix multiplication algorithm described by Lee and Seung (1999), which constrains the temporal com- ponents and weighting coefficients to be non-negative. The rational for application of the NMF was that our data consisted of non-negative values (rectified EMG activity) and we found negative weightings using the FA (Ivanenko et al., 2003), whereas the NMF constraint allows only additive and not subtractive combinations. We reduced the dimensionality of the data to the same final number of temporal components as in the FA for both the ICA and the NMF analysis. This is reasonable because the total variance explained by temporal components was similar and because we could compare the same number of temporal components extracted by the three methods.

Theoretically, EMG waveforms can be analyzed in different units (in microvolts, normalized to the maximal value across conditions, to the EMG level during maximal voluntary contraction, to the functional cross-sectional area of the muscle or to the number of motor neurons of a particular muscle in the spinal cord, etc.). Using the correlation matrix for the FA (see above) automatically implies normalization of each EMG waveform to its SD value. To compare the results of the FA with those of the ICA and NMF, the amplitude of EMG waveforms was normalized by their mean SD values before the application of the ICA and NMF. This is reasonable because we were mainly interested in rhythmic patterning elements in the control of locomotion (temporal components) rather than in muscle synergies (weighting coefficients). Nevertheless, we also applied the ICA and NMF to the non-normalized (in microvolts) EMG data sets to verify whether a normalization procedure might considerably normal walk
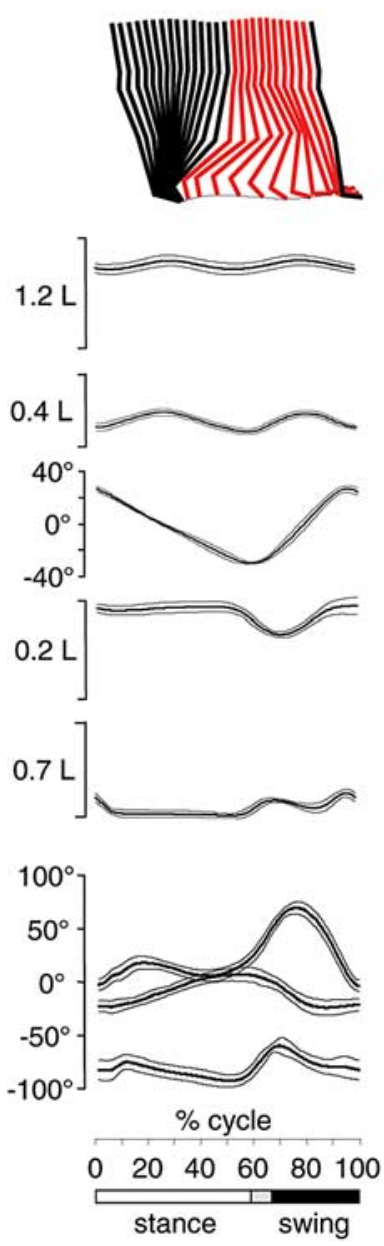

kick
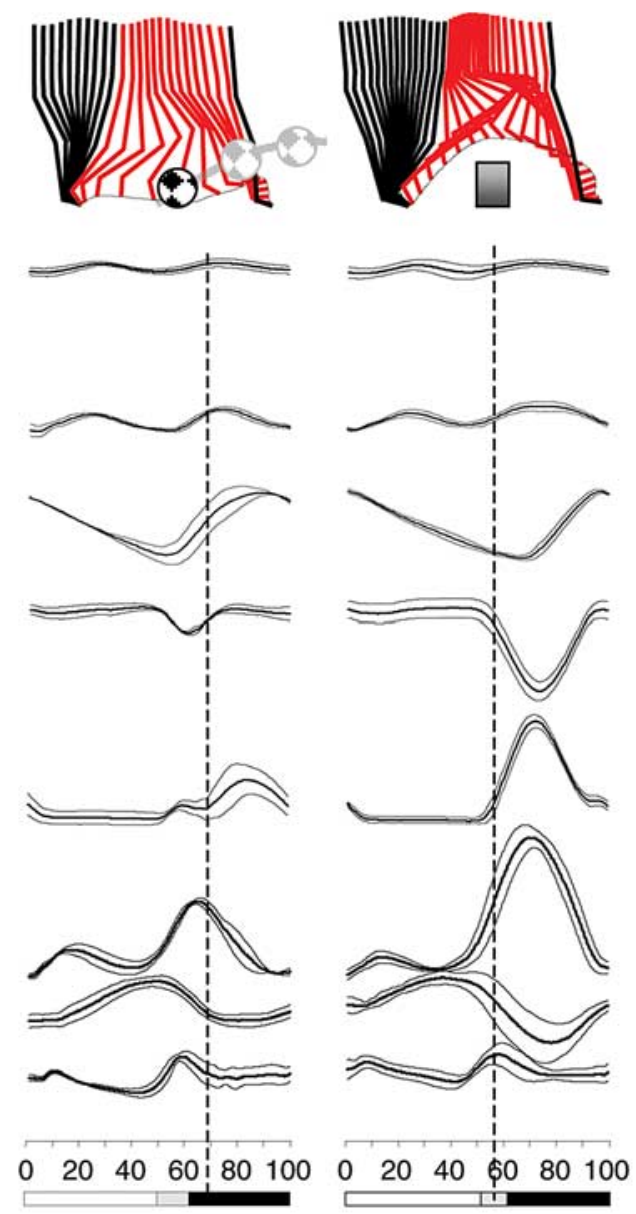

obstacle
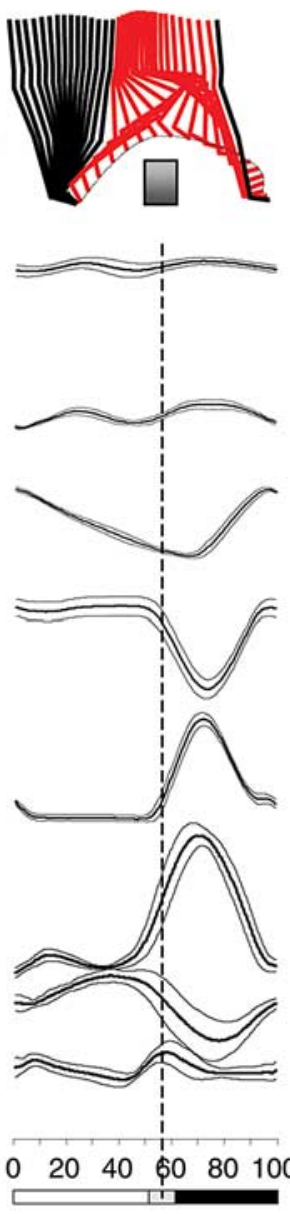

stoop right
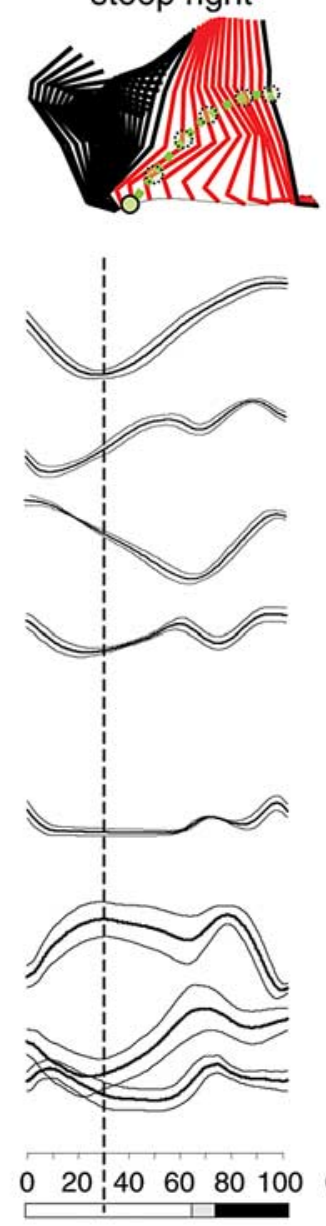

stoop left
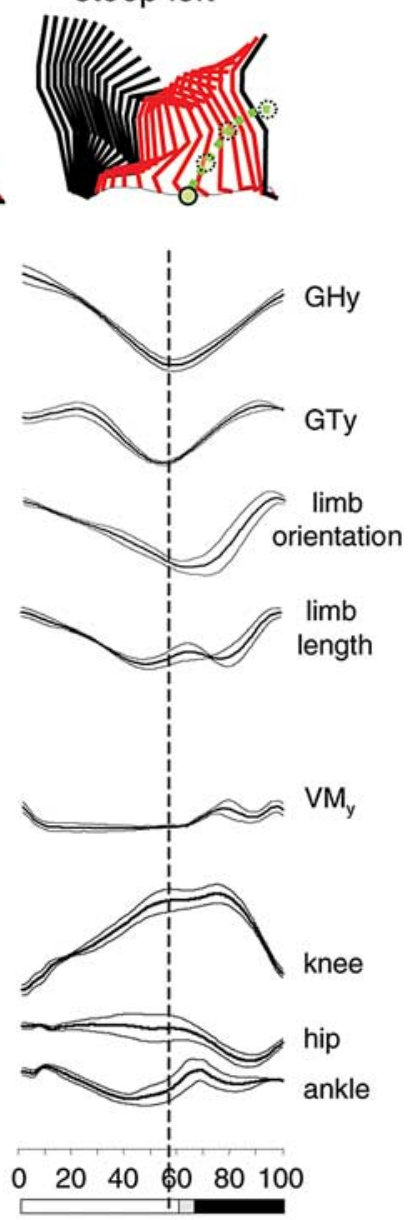

Figure 1. Averaged trunk and right limb kinematics ( 8 subjects) in the normal walk, kick, obstacle, stoop-right, and stoop-left conditions. From top to bottom, stick diagrams, normalized vertical shoulder displacements $\left(\mathrm{GH}_{\mathrm{y}}\right)$, vertical hip displacements $\left(\mathrm{GT}_{\mathrm{y}}\right)$, limb orientation, limb length, vertical foot displacements $\left(\mathrm{VM}_{\mathrm{y}}\right)$, and knee, hip, and ankle joint angles $\pm \mathrm{SD}$ are shown. $\mathrm{L}$, Limb length (thigh plus shank). As the relative duration of stance varied, a hatched region indicates an amount of variability in the stance phase duration across subjects. Dotted vertical lines correspond to the timing of the voluntary task. 
$A$

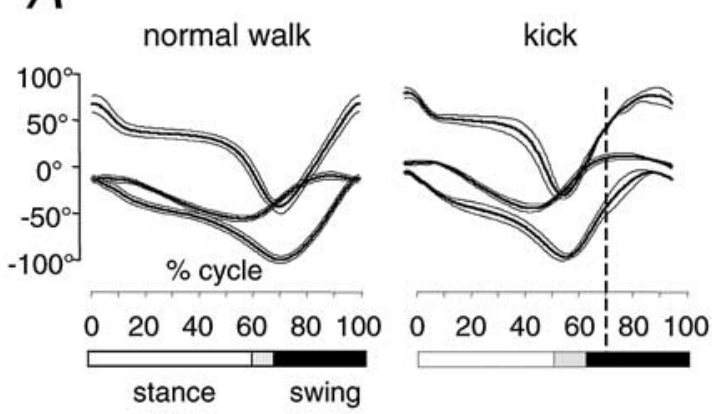

$B$
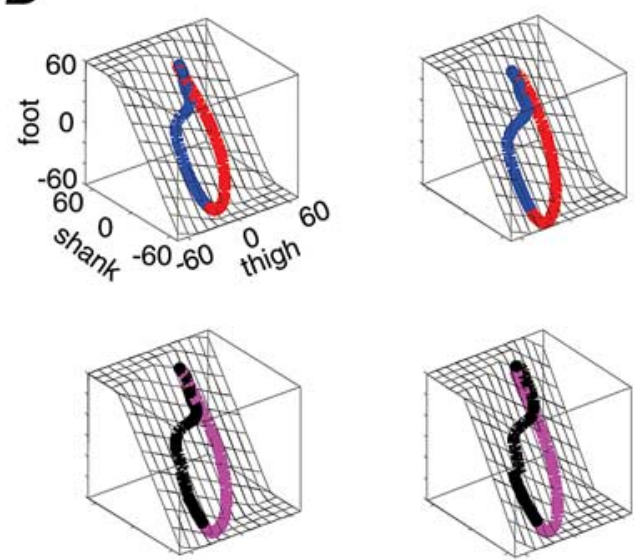

Right leg

obstacle
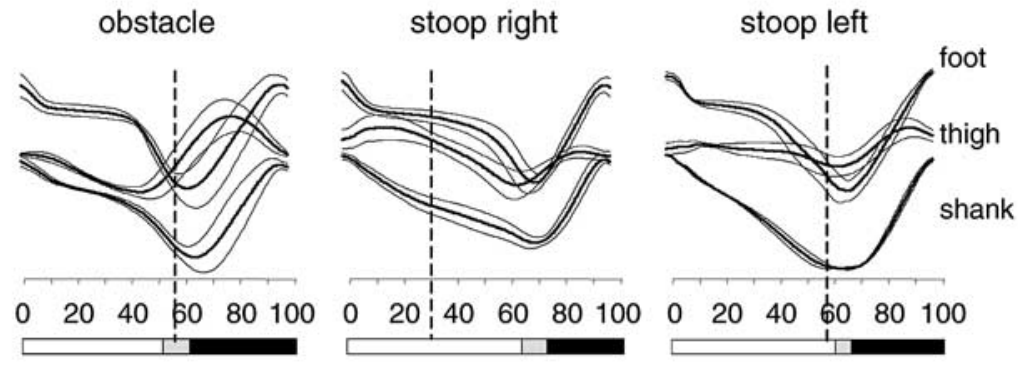

Right leg
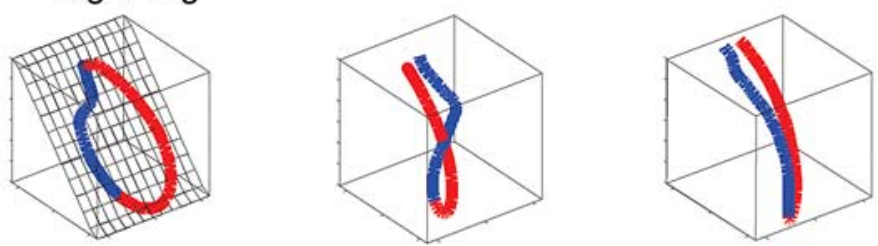

Left leg
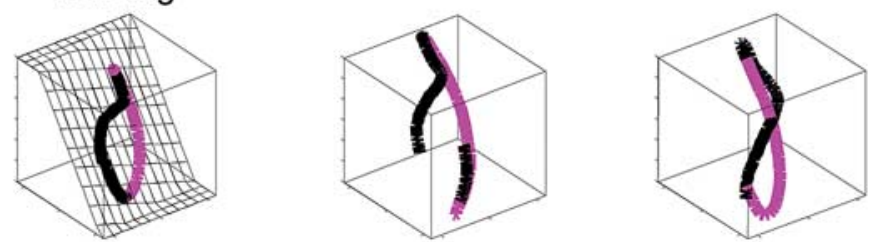

Figure 2. Intersegmental coordination. $A$, Ensemble averages \pm SD ( $n=8$ subjects) of thigh, shank, and foot elevation angles of the right leg. $B$, Corresponding gait loops plotted in the third dimension both for the right and left legs. A loop represents one gait cycle defined for the right leg (time interval between the two consecutive heel strikes of the right leg, so that the beginning of the cycle was not equivalent for the left leg) and is obtained by plotting the thigh waveform versus the shank and foot waveforms (after mean values subtraction). Paths progress in time in the counterclockwise direction, with the foot strike and lift-off corresponding approximately to the top and bottom of the loop, respectively (the swing phase is in red and in magenta for the right and left legs, respectively). The interpolation planes result from orthogonal planar regression. For stoop conditions, the interpolation planes are not shown because there were significant deviations from the plane during the stance phase for both legs. Note a similar plane orientation in the normal walk, kick, and obstacle conditions.

affect the shape of temporal components and a comparison of loadings across conditions.

Although similar in their idea of linear combination of basic components, these algorithms can differ in their ability to identify basic temporal components and corresponding weighting coefficients across muscles. They can be differently sensitive to noise or be constrained to only non-negative values (as in the case of NMF). Therefore, by using different statistical methods, we verified whether the presence of rhythmic patterning elements is a consistent feature or is strongly affected by a particular algorithm or constraint.

\section{Statistics}

A PCA was performed using Statistica version 6.0 (StatSoft, Tulsa, OK). Statistical analyses (Student's $t$ tests and ANOVA) were used when appropriate. Reported results are considered significant for $p<0.05$. To compare loadings and activation wave shapes, simple linear correlations ( $r$ values) of loadings and activation waveforms were calculated and are presented throughout, except where stated otherwise. Statistics on correlation coefficients was performed on the normally distributed, Z-transformed values. Spherical statistics on directional data were used to characterize the mean orientation of the normal to the covariation plane (see above) and its variability across steps, subjects, or conditions.

\section{Results}

\section{Kinematics patterns}

We recorded body and limb kinematics and ground reaction forces in eight volunteer subjects while they walked over ground at $\sim 5 \mathrm{~km} \mathrm{~h}^{-1}$ (Table 2). In addition to normal locomotion, each subject also performed four voluntary tasks during a locomotion cycle in four separate sets of trials. Figure 1 shows the right leg kinematics for each task compared with the kinematics of normal walking. The task kinematics of the kick, in which the subjects kicked a ball placed in the locomotion path, were most similar to those of normal locomotion. Most of the kinematics differences occurred in the latter part of the swing phase after the ball was kicked. Nevertheless, the joint-angle trajectories were nearly identical to those of normal walking. The limb axis trajectories, both limb axis orientation, and length were also quite similar to those of normal walking (Fig. 2A). Finally, the limb trajectory in the limb segment angle space was very similar to that of normal walking (Fig. $2 \mathrm{~B}$ ), and it was mostly confined to a plane (Borghese et al., 1996). The planarity of the trajectory was quantified by the percentage of VAF by the first three eigenvectors $\left(\mathrm{PV}_{1}, \mathrm{PV}_{2}\right.$, and $\mathrm{PV}_{3}$ ) of the data covariance matrix. For a plane, $\mathrm{PV}_{1}>$ $\mathrm{PV}_{2} \gg \mathrm{PV}_{3}$ and $\mathrm{PV}_{3}$ should be close to 0 (for a line, both $\mathrm{PV}_{2}$ and $\mathrm{PV}_{3}$ should be close to 0 ). For the kick task, $\mathrm{PV}_{2}$ was $15.1 \pm 6.1 \%$ and $\mathrm{PV}_{3}$ was $1.0 \pm 0.3 \%$.

When the subjects were required to step over an obstacle placed in their path, the kinematics were altered by a much greater shortening of the limb during swing, and a correspondingly greater excursion of the knee and thigh segment angles (Patla et al., 1991; McFadyen and Carnahan, 1997; van Hedel et al., 2002). The limb axis trajectory was correspondingly different 


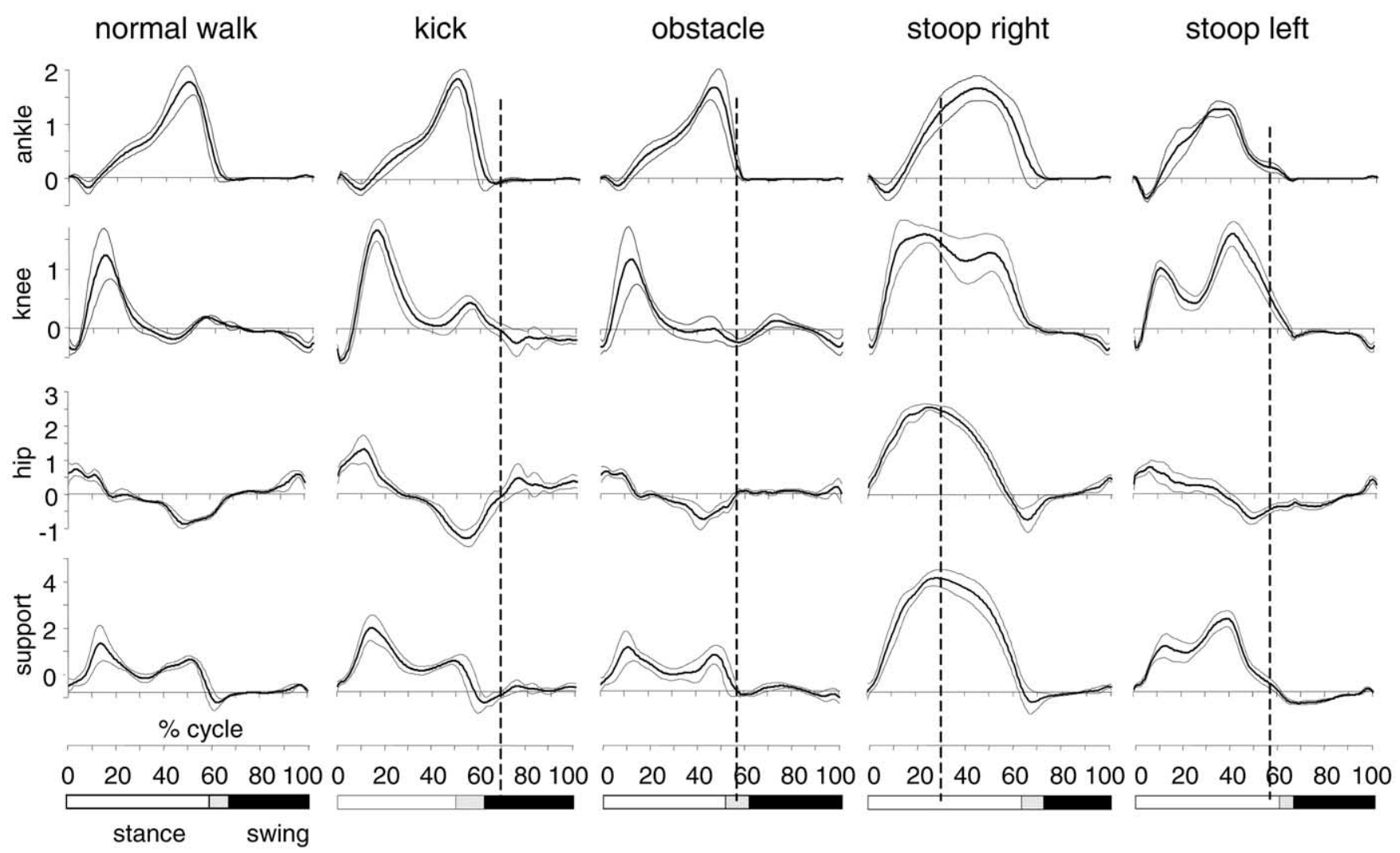

Figure 3. Ensemble-averaged ankle, knee, hip, and support moments of force $\left(\mathrm{Nm} \mathrm{kg}^{-1}\right)$ of the right leg in the five walking conditions.

for limb axis length, but orientation was similar to that of normal walking (Fig. 1). The higher step widens the limb trajectory in the segment angle space $\left(\mathrm{PV}_{2}=18 \pm 8 \%, \mathrm{PV}_{3}=0.7 \pm 0.3 \%\right)$ along the axis of the thigh segment angle (Fig. $2 B$ ). Otherwise, it remains in a similar plane as the trajectory during normal walking: the mean orientation of the normal to the plane (the third eigenvector of the covariance matrix) differed from that of normal walking by $14^{\circ}$, the angular SD in the plane orientation (called spherical angular dispersion) being $8^{\circ}$. The left limb segment angle trajectory during the same step cycle is almost indistinguishable from the normal walking trajectory.

The greatest overall changes from normal walking kinematics occurred when an object was grasped from the floor during locomotion. In these cases, the hip and knee angle trajectories were quite different, although the segment angle trajectories were more similar to those of normal walking (Fig. $2 A$ ). However, the limb axis length trajectories were also different in the stoop tasks (Fig. 1). The limb length during normal walking was mostly constant during stance with a single shortening phase during swing. In the stoop tasks, the limb shortens during the stance phase as the body is crouched down. For stoop right, there is an additional shortening during swing resulting in two cycles of shortening in the same gait cycle. For stoop left, the shortening in stance is mostly continued into the swing phase with a single cycle of shortening in the gait cycle that has a longer than normal duration.

The movement trajectory in limb segment angle space (Fig. $2 B)$ was no longer clearly planar in these stooping tasks $\left(\mathrm{PV}_{2}=\right.$ $7.8 \pm 1.7 \%$ and $\mathrm{PV}_{3}=3.8 \pm 2.4 \%$ for stoop right; $\mathrm{PV}_{2}=2.9 \pm$ $1.3 \%$ and $\mathrm{PV}_{3}=1.5 \pm 0.9 \%$ for stoop left). Moreover, these tasks required asymmetrical body movements that produced quite different limb segment angle trajectories on the two sides of the body. In all cases, the deviations from the normal walking plane were observed mainly during the stance phase (blue or black traces) because the movement trajectory during the swing phase remained in a plane similar to that of normal walking. Moreover, the trajectories of the right leg during the stoop right and of the left leg during the stoop left were similar and tended to be confined to two different planes, one for swing and another for stance. The respective trajectories for the opposite side were basically linear in this representation (i.e., one-dimensional instead of two-dimensional, because $\mathrm{PV}_{2}$ was $\sim 3 \%$, on average).

\section{Kinetics patterns}

The joint-angle and support moments of the right leg were all quite similar in normal locomotion and for the kick and obstacle tasks (Fig. 3) (McFadyen and Winter, 1991; Chou and Draganich, 1997; McFadyen and Carnahan, 1997). Note that kick force was limited in our experiments (see Materials and Methods). However, there were significant differences from normal walking in most of these variables during the stoop tasks. The stoop right showed significant increases in the joint-angle moments at all three joints, occurring early in the cycle when the body is lowered for the grasp. A similar difference is also seen in the support moment. A similar increase in moment occurred in the latter part of the stance phase in the stoop-left task. Thus, it is clear that joint torques and muscle forces were also very different from those of normal walking in the two stoop tasks.

\section{Muscle activity patterns}

We recorded EMG activity from 16 leg and trunk muscles in the eight subjects and additional 15 neck, arm, leg, and trunk muscles in three to five of the subjects during most of the task conditions (see Materials and Methods) (Table 1). The average EMG re- 


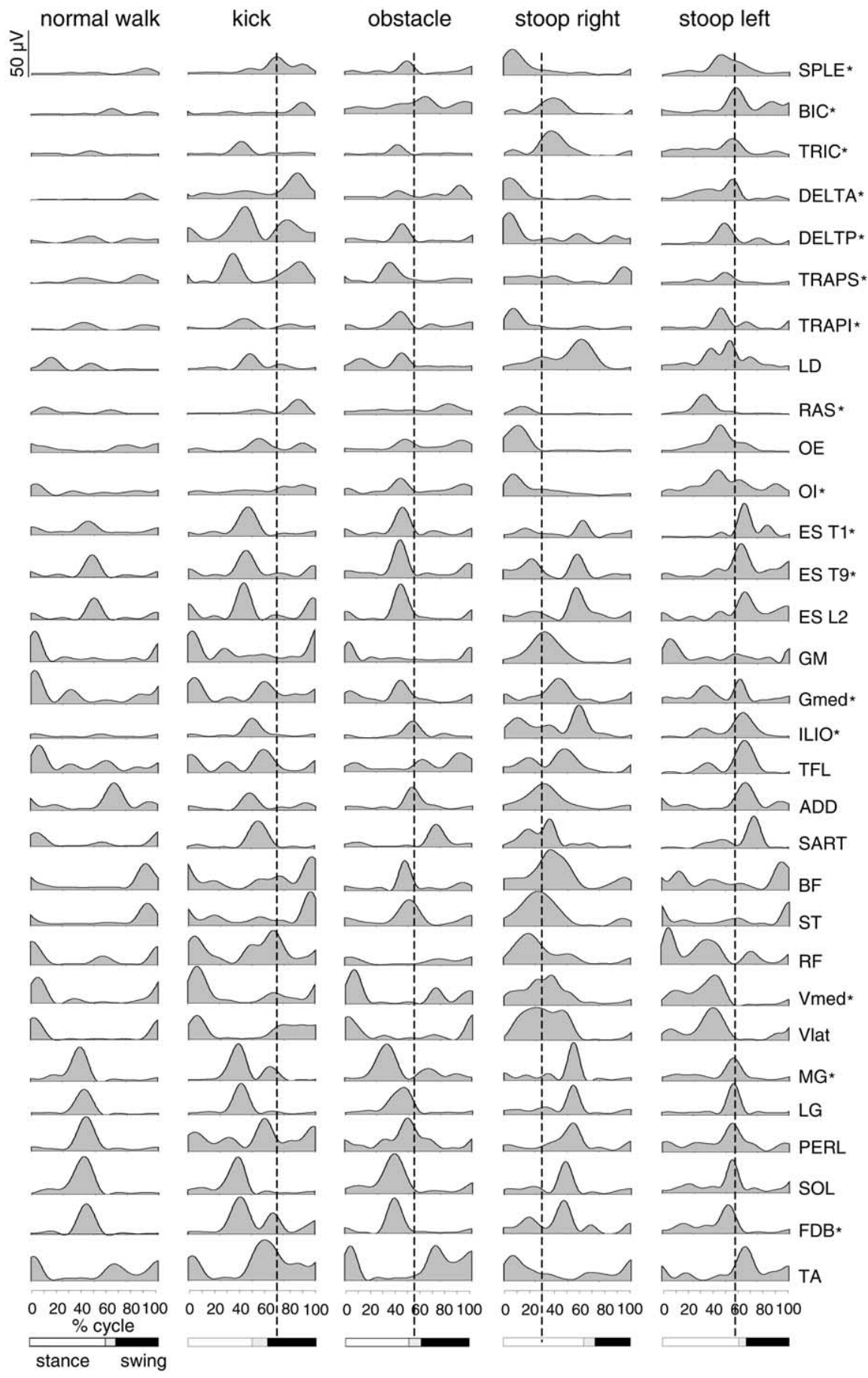

Figure 4. Ensemble-averaged activity patterns recorded from eight subjects for 31 muscles. EMG records were filtered with a low-pass cutoff of $5 \mathrm{~Hz}$. Asterisks indicate EMG patterns obtained from less than eight subjects.

corded from each active muscle across subjects is illustrated in Figure 4 . The activity varied significantly from task to task for most of the muscles recorded. We found that both amplitudes and patterns of activity were altered by these tasks. In some cases, activation patterns were completely different from those of normal walking (for example, Vlat, Sol, and SART in the stoop tasks), whereas in others the patterns were basically the same but with an additional activation component (for example, BF, ST, or ADD during the obstacle task) (McFadyen and Winter, 1991; Patla et al., 1991). The patterns of activation across muscles that were most similar to normal walking were the patterns observed in the locomotion. kick and obstacle tasks $(r=0.65 \pm 0.21$ and $0.64 \pm 0.15$, respectively). The patterns were quite different during the stooping tasks $(r<0.2)$.

We recorded surface and intramuscular EMGs simultaneously in seven leg muscles of one subject to control the effectiveness of cross-talk rejection by our surface recordings during the normal walking, obstacle, and stoop-right conditions. The surface EMGs were generally well correlated with the intramuscular EMGs in all conditions (Ivanenko et al., 2004). The mean correlation coefficient between intramuscular and surface EMGs across all seven muscles and conditions was $0.87 \pm 0.13$.

\section{Comparison of analytical methods}

In our previous study (Ivanenko et al., 2004), we used a PCA FA to find common components in the EMG patterns across muscles. Other statistical approaches have also been successful in extracting independent components from data such as the EMG patterns (e.g., ICA; see Materials and Methods). Because they all place different restrictions on the outcomes, they may also extract different sets of components. Thus, we begin here by comparing results from three different statistical methods (FA, ICA, and NMF) to analyze the same set of averaged normalized EMG waveforms (plotted in Fig. 4) in the normal walk condition. The ordering of the ICA and NMF components was matched in such a way that they showed the highest correlation with corresponding FA factors (Fig. 5). The basic temporal components computed using the ICA were generally quite similar to the FA components (mean correlation $r=0.91 \pm 0.08)$. In addition, the loadings or weighting coefficients on particular muscles were also similar in the two methods (Fig. 5B): a loading pattern across 31 muscles was correlated with the loading pattern of the FA varimax factors ( $r=0.87 \pm 0.13$ for all components). Not surprisingly, the component clusters defined by the ICA may have much in common with other activity recognition techniques (Makeig et al., 2004), at least as far as it concerns rhythmic patterning elements in the human

It is also of interest that the NMF method gave comparable results despite the fact that it constrained the analysis to only non-negative temporal components and non-negative weighting coefficients. The NMF was developed to analyze frog EMGs, which can have long silent periods with a few episodes of activity. Instead, our records more often showed a level of activity that was modulated up or down. Nevertheless, the NMF method gave a comparable structure of the basic temporal activation components (mean correlation $r=0.92 \pm 0.10$ ) (Fig. 5A), and the 


\section{normal walk}
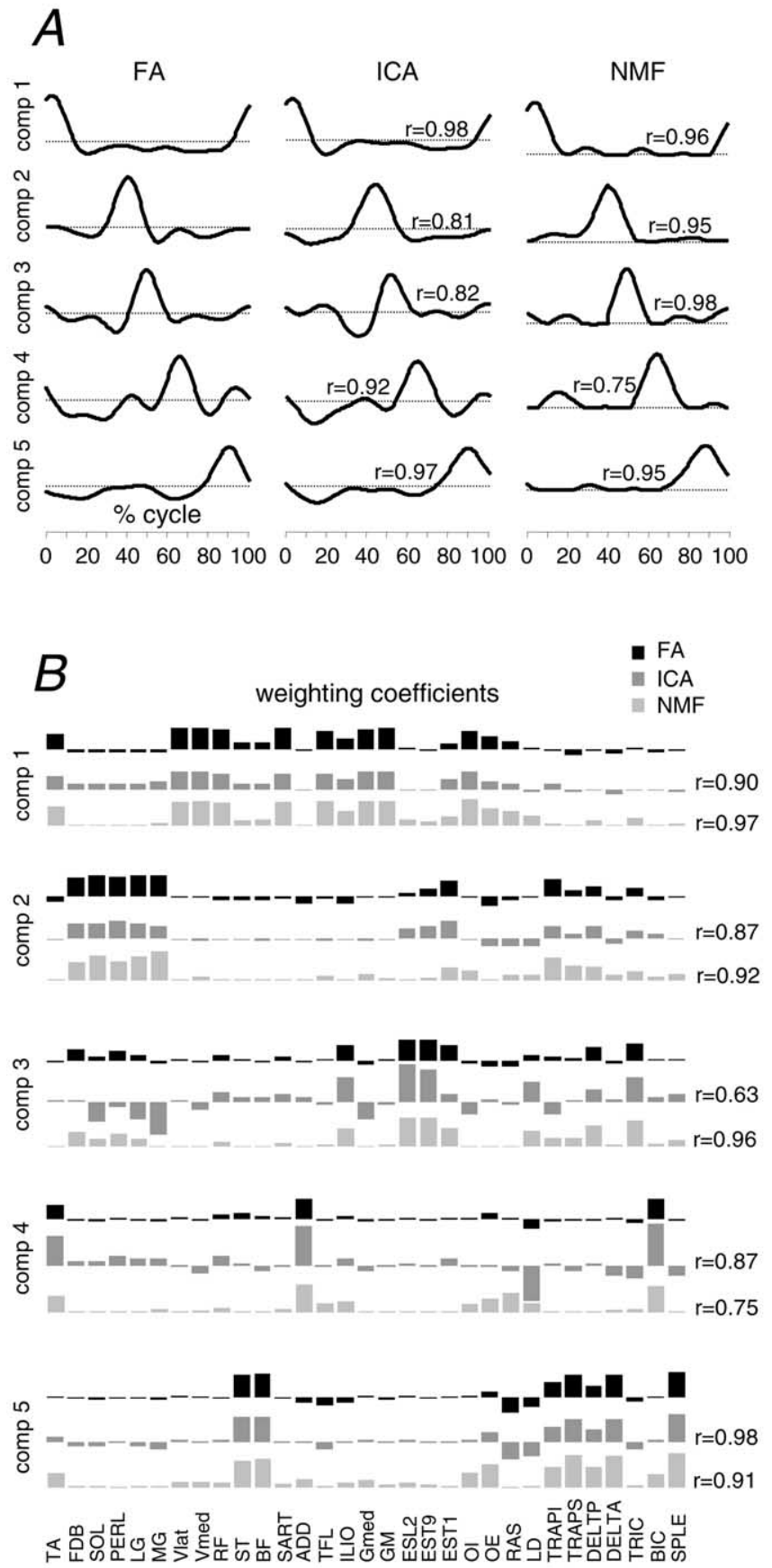

Figure 5. Comparison of activation components $(\boldsymbol{A})$ and corresponding weighting coefficients (loadings) across 31 muscles (B) obtained using different statistical methods (FA, ICA, and NMF) in the normal walk condition. The amplitude of EMG waveforms was normalized by the mean SD before application of the ICA and NMF. $A$, Correlations between components obtained from the ICA and NMF and corresponding varimax factors (FA). $\boldsymbol{B}$, Loadings for each muscle listed across the bottom are compared for each analysis method (black and gray bars). The correlation of weighting patterns with respect to the FA is shown at the right of each record. comp, Component.

loading patterns were also similar (the mean correlation across components was $0.91 \pm 0.07)$. In those cases in which the factor loading was negative for the FA or ICA (e.g., component 2 for OE or component 3 for Gmed), the loading tended to be zero for the NMF components. It is also worth noting that the profile of temporal components in the ICA and NMF did not depend signifi- cantly on the normalization method (i.e., when we analyzed EMGs in microvolts: mean correlation to the FA components $r=$ $0.93 \pm 0.09)$. Finally, the total variance explained by basic temporal components was also similar. For instance, in Figure 5, the percentage of variance explained by all components during normal walking was $90 \%$ using the FA, $88 \%$ using the NMF, and $92 \%$ using the ICA.

In summary, the different algorithms seem to converge to a similar solution about the temporal structure of the EMG activity pattern during human locomotion although they are based on different sets of assumptions. The common temporal patterning elements each consist of a relatively narrow peak of activation at a specific timing point in the gait cycle.

\section{Factor analysis}

Given the nearly equivalent results of the previous section, we selected the FA approach to find common components in the EMG patterns across muscles to be consistent with previously published results (Davis and Vaughan, 1993; Olree and Vaughan, 1995; Ivanenko et al., 2003, 2004). We analyzed EMG patterns separately for each subject and for each task (Fig. 6A). The basic result was that the five temporal components (or factors) found for normal walk were common to all of the tasks across subjects. Their similarity across tasks is illustrated by a plot of their peak phases (Fig. $6 \mathrm{~B}$ ) and peak widths (Fig. 6C) across tasks. The average differences in peak phases were less than $\pm 5 \%$ of the cycle, with the exception of component 4 during the kick task (Fig. 6B). This component was consistently phase advanced in this task, and that is likely because the stance phase was consistently shorter in this task $(55.5 \pm 3.4 \%$ of the cycle compared with $64.6 \pm 1.6 \%$ in normal walking) (Ivanenko et al., 2004). The width of the main peak estimated as FWHM was relatively invariant across components and conditions, with the one exception of the sixth component in grasp right, which tended to be wider than in all other tasks (Fig. 6C and see below).

The mean correlation of individual components with averaged normal walk temporal components was $0.76 \pm 0.14$ across all components and conditions. Because the timing could vary slightly from subject to subject (Fig. 6A), we also shifted the phases slightly for best peak alignment (within $\pm 5 \%$ of the cycle) and found the pattern correlations were even higher $(0.87 \pm 0.09)$.

These are essentially the same five temporal components reported previously for normal locomotion at different speeds and locomotion with body weight support (Ivanenko et al., 2004). They were the only significant components observed in normal walking, accounting for $90-95 \%$ of the EMG pattern variance across muscles. Higher-order temporal components (that explained, on average, only $3.1 \pm 1.1 \%$ of variance) displayed very high variability across subjects and conditions (Fig. $6 \mathrm{~A}$, comp 7 ) and were therefore considered to be nonsignificant and consequently dropped from the analysis. In each voluntary movement task, there were six significant components, in which the additional component was associated with an element of the task. Once again, this was not a property of the FA approach because we obtained the same basic result with the other analytical methods (Fig. 7).

The sixth component that became significant for the kick has a peak occurring during the kick, and it seems to be associated with trunk muscle activity that may stabilize the body. For the obstacle, the sixth component occurred at lift-off and was mostly associated with proximal limb muscles. The two stoop tasks had a sixth component with its peak corresponding to the timing of the stoop. This component was associated with both trunk and prox- 

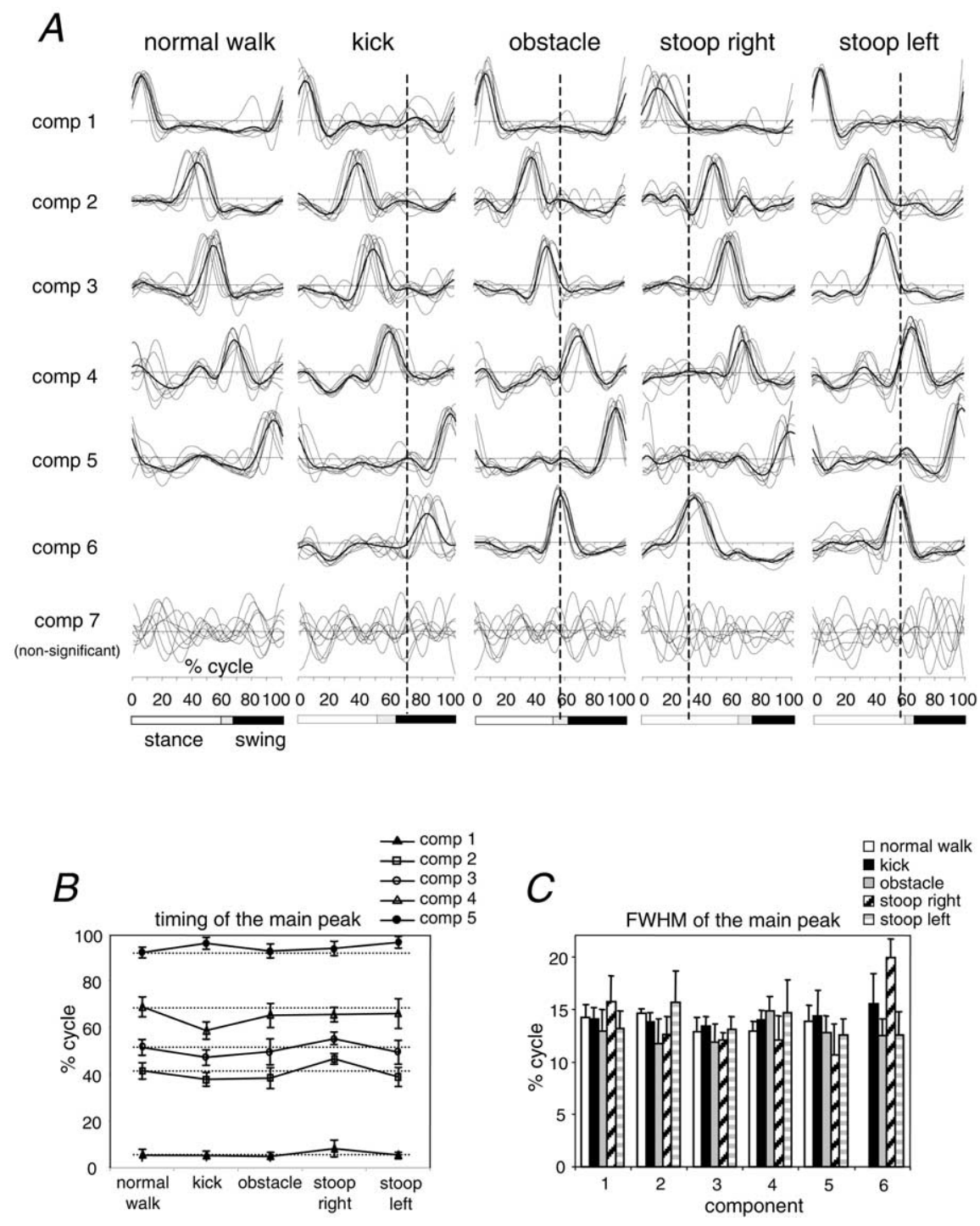

Figure 6. Activation components by subject. $A$, Varimax factors derived from muscle activity patterns by the FA for each of eight subjects (thin lines). Bold traces show the average of the components across eight subjects. Five common components across conditions are located in "chronological" order (with respect to the timing of the main peak), whereas the new sixth component in the voluntary tasks was plotted separately. The next higher-order component (that explained, on average, only $\sim 3 \%$ of variance) was also plotted. It displayed very high variability across subjects and conditions and therefore was dropped from the analysis. Stance phase was somewhat different across subjects as indicated by hatched area of the stance-swing bar. $\boldsymbol{B}$, The timing $\pm S D$ of the peaks of five common components across tasks. The five basic components are each characterized by a relatively narrow peak of activation at a particular phase of the cycle. Dotted horizontal lines correspond to the mean values during normal walk. C, The width + SD of the main peaks of components across tasks was estimated as the FWHM. comp, Component.

imal leg muscle activation in both cases. The sixth component for stoop left and obstacle were similar in phase (the onset of swing), perhaps because they both correspond to the appearance of a prominent vertical component of movement at the onset of swing that includes the onset of the trunk lift and foot lift (Fig. 1).

We compared the average of each temporal component across subjects with the components obtained from a FA that included all subjects for each task (Fig. 8A). The similarity of the results from these two analyses is consistent with the small betweensubject variability we observed. In the few cases in which the correspondence was not as good, the differences might be attributable to some variability in the way different subjects performed the tasks, such as in the relative stance duration, or to an uncertainty in identifying components with low loadings [i.e., when the component did not account for much of the total variance (e.g., compo- nents 4 and 5 in the stoop-right and stoopleft tasks)]. In the voluntary tasks, the six components accounted for $89-95 \%$ of the variance in EMG patterns for all of the active muscles recorded. For the stooping tasks, this included six arm muscles for five of the subjects. The results from those subjects are indistinguishable from the results obtained from only the leg and trunk muscles (although the addition/removal of EMG variables may have had some effect on loadings and/or percentage of VAF by each component).

\section{Treadmill locomotion with constant belt acceleration and deceleration}

Although we showed previously that the components are relatively invariant across different speeds (Ivanenko et al., 2004), we did not study whether the components remain similar when the speed is not constant. We considered here the possibility that a new component might reflect changes in locomotion speed. For example, peak-to-peak speed oscillations over a cycle were greater during the stoop than during normal walking by $0.4-0.7 \mathrm{~km}$ $\mathrm{h}^{-1}$ (Table 2). To control for this speed variation, we recorded EMG patterns during changes in treadmill speed (see Materials and Methods) that generally corresponded to the changes occurring during gait perturbations. We found that the components derived for single steps that spanned $5 \mathrm{~km} \mathrm{~h}^{-1}$ in treadmill acceleration or deceleration were indistinguishable from the corresponding components determined for a constant speed of $5 \mathrm{~km} \mathrm{~h}^{-1}$.

\section{Percentage of variance explained}

Note that we ordered the components here according to the timing of the peak rather than according to the rank VAF as we did in our previous study (Davis and Vaughan, 1993; Ivanenko et al., 2003, 2004). Therefore components 1 and 2 are presented in the reverse order of what we reported previously, and component 5 is the previous component 3. Component 4 typically accounted for smaller variance than any other component during normal walking (Fig. 8 B). By comparing the VAF by each component in normal walking with those for each task, we can observe that although the five basic components may have been the same across tasks, the weights were not. In particular, component 4 , which occurs just before the kick, accounts for more variance in the kick task (21\% vs $11 \%$ ) (Fig. 8 B, left). Component 3, occurring just before the leg lift, is heavily weighted in normal walking and similarly weighted in the obstacle task, but component 6, which occurs between components 3 and 4 , is also heavily weighted. Component 1 , occurring just after touch down accounts for most of the variance during normal walking, but it accounts for approximately onehalf that amount during the kick and obstacle tasks, because of 
$A$
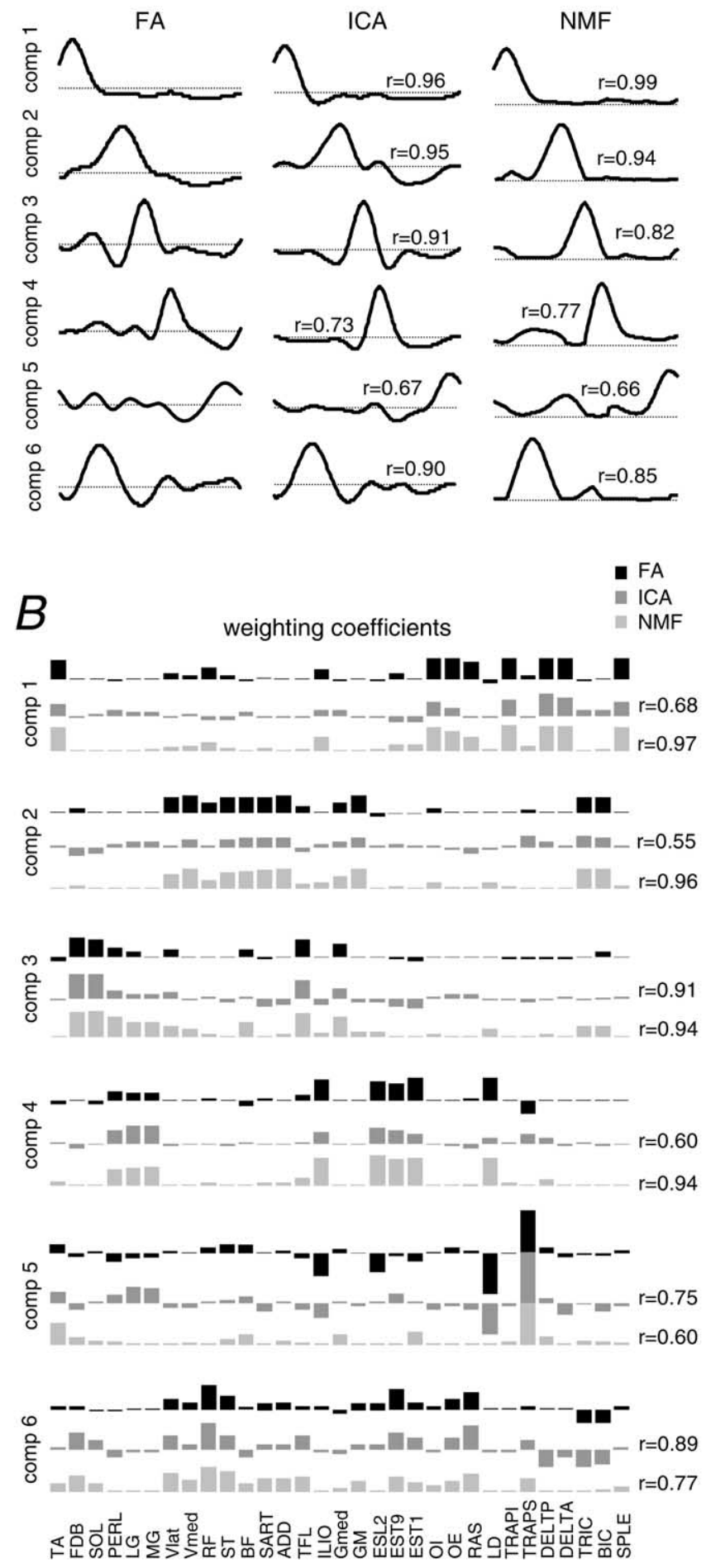

Figure 7. Comparison of activation components $(\boldsymbol{A})$ and corresponding weighting coefficients ( $\boldsymbol{B}$ ) in the stoop-right task using different statistical methods (FA, ICA, and NMF) (see Fig. 5 for details). comp, Component.

the greater VAF relative to normal walking of the other component.

The VAF was also different for the stoop tasks in which the sixth or additional component was heavily weighted compared with the basic five components (Fig. $8 B$, right). We also found that the five components were weighted differently in the two versions of this task. The VAFs were very low for components 3 and 4 in the stoop-right task, whereas they were quite high in the left stoop, in which component 1 and 2 VAFs were very low (Fig. $8 B$, right).

\section{Weighting coefficients (across muscles) of five basic temporal components}

The relative strength of the effect of each activation component on a given EMG pattern is given by the component "loading" or "weighting coefficient" (Figs. 5B, 7B). Changes in the weighting coefficients of the five basic temporal components in all voluntary tasks relative to the normal walk were estimated by correlating a set of weighting coefficients (across 31 muscles) for each component with a corresponding set in the normal walk task (Fig. 9). There was a fairly large consistency of relatively high correlation coefficients (Fig. 9, filled bars) across different statistical methods (FA, ICA, and NMF), revealing a similar set of muscles primarily involved in this basic muscle activation. In general, weighting coefficients (loadings) were more affected for components close to the timing of the voluntary task (Fig. 9, open bars). For instance, component 4, which occurred just before the kick, accounted both for a stronger activation of some muscles (TA, SART, ILIO) (Fig. 4) as well as for the recruitment of additional muscles (i.e., PERL, MG, RF, Vmed, FDB) appropriate for the kick force. This changes the balance of muscles represented by this component. Likewise, it is also reasonable that neighboring components in the stoop tasks (i.e., components 2-4) are loaded differently, because of a reorganization of muscle activations needed to unbend and lift the trunk after the grasp (Fig. 1). Moreover, it is worth noting that the correlations with normal walk loadings showed qualitatively the same trend if the statistical analyses (ICA and NMF) were performed on the non-normalized (in microvolts) EMG waveforms (the results were similar to those presented in Fig. 9). This analysis (when we used EMGs in microvolts) showed some relative redistribution of weighting coefficients across muscles, but it did not improve overall correlations of loading vectors across conditions; positive correlations even slightly decreased (by $\sim 7 \%$, on average). That is, the normalization was not responsible for the differences in component loadings we observed across tasks.

Finally, despite the similarity of the five basic components across subjects (Fig. 6A), the individual loadings were somewhat less similar because there were definite individual differences. For instance, the loadings were highly correlated across subjects for all tasks and components, $0.88+0.09$ overall (this characterizes correlations of individual loadings with those extracted from the analysis performed on ensemble averaged EMGs) (Fig. 4); however, there were a few individual differences that were significant. The range of correlation coefficients was $0.32-0.99$. Such differences were also evident during normal walking (Winter and Yack, 1987; Ivanenko et al., 2004) and could be related to differences in the individual anthropometrical characteristics (modified biomechanical requirements on muscle activations), slightly different kinematics, or EMG electrode placements.

In summary, although the intrinsic temporal structure of the locomotor program (activation timings) was conserved across conditions, we often observed a different balance of limb and trunk muscles associated with each component.

\section{Walking versus standing}

To help clarify the nature of a sixth component with distinct timing in the voluntary tasks, we also asked subjects to perform 


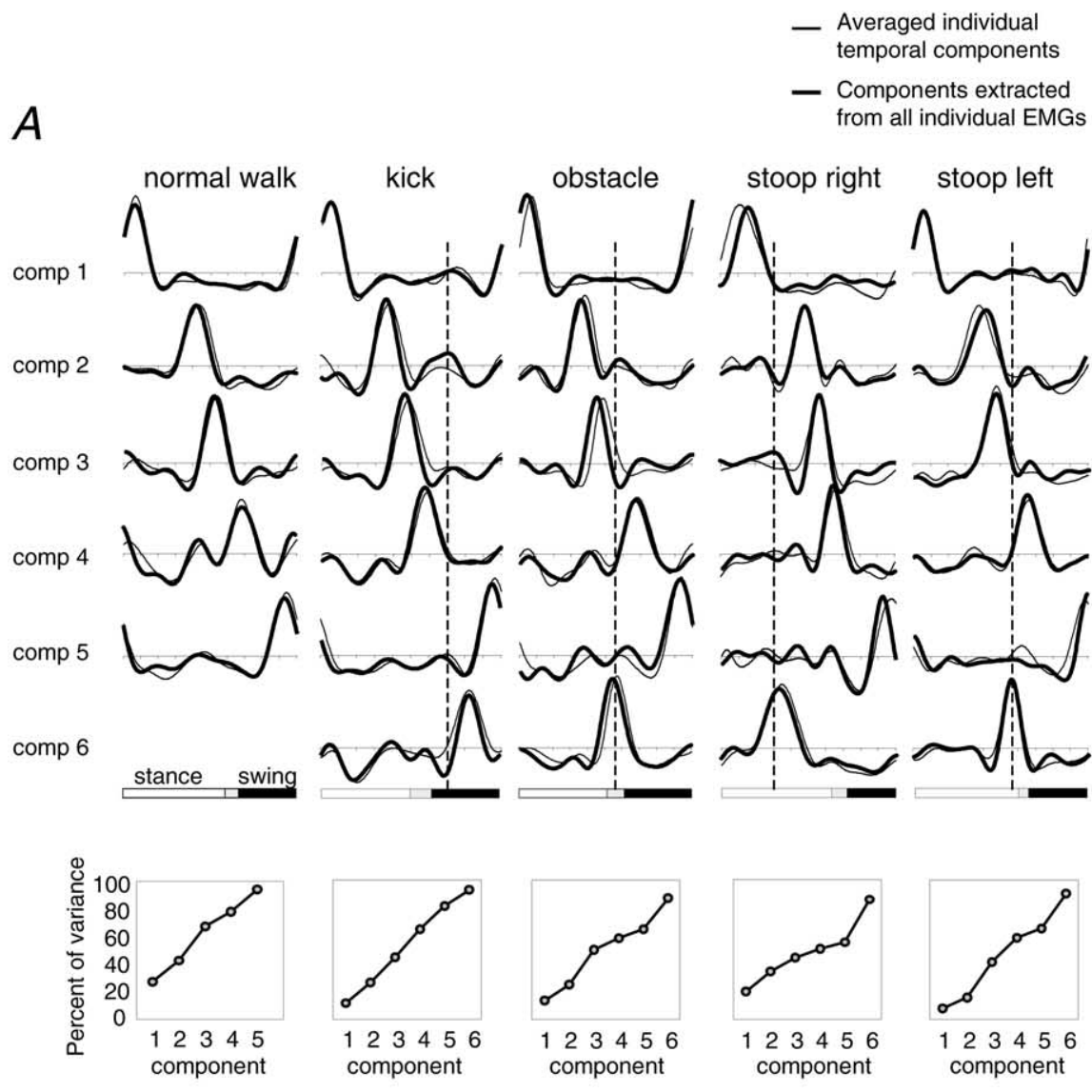

$B$

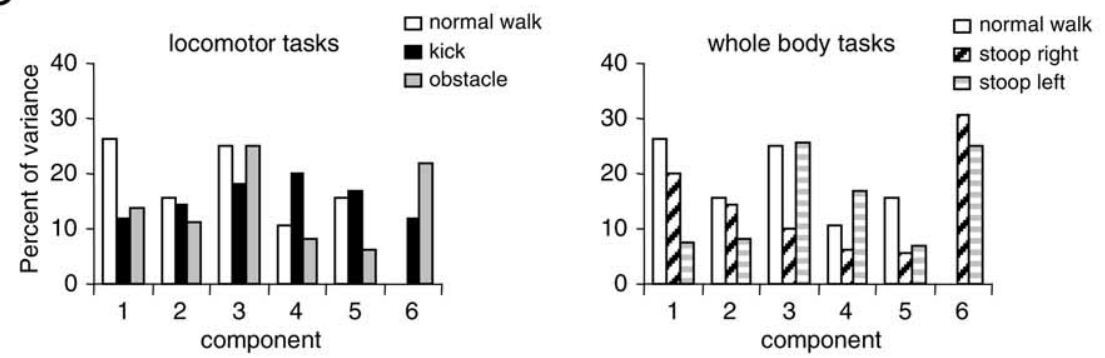

Figure 8. Averaged activation components. A, Averaged FA components (comp; black traces; same as in Fig. 6) are superimposed to those derived from all individual muscle activity patterns pooled together (thick gray traces). The cumulative percentage of variance explained by each component in the latter case is shown for each task in the bottom panels. $\boldsymbol{B}$, Percentage of variance explained by each component in the data sets obtained from all individual muscles (same as in $\boldsymbol{A}$; bottom panels). Locomotor tasks (kick and obstacle; left) and whole-body tasks (stoop right and left; right) are shown separately.

some of the voluntary tasks in place, (i.e., without locomotion) (see Materials and Methods). We examined two cases, stoop-inplace and step-in-place, in which the subject either reached down and stood up or produced a high step with the right leg, both in the same cadence used for the stoop or obstacle tasks during locomotion. If the new component in the voluntary tasks (Fig. $6 \mathrm{~A}$ ) were a result of a specific voluntary component added to the locomotion, we might find a component similar to the sixth component with similar muscle loadings for the in-place tasks. If, however, the two tasks (e.g., stoop-in-place and stoop during locomotion) were unrelated from a control perspective, we would expect different results.
The stoop-in-place task (Fig. 10A) revealed two components that accounted for $>60 \%$ of the variance, with the higherorder components being variable from trial to trial. One of the components (Fig. $10 \mathrm{~A}$, comp 2) had a loading pattern across 29 muscles that was correlated with the pattern observed for the same subjects for component 6 in the stoop-right task ( $r=$ $0.61 ; 0.69$ when just the 15 leg muscles are considered) (Fig. 10A, bottom panel). Both components were loaded primarily on leg and trunk muscles (Vlat, RF, ST, BF, SART, ADD, Gmed, GM, ESL2, EST9, and EST1) rather than on arm muscles. A few muscles (LG, Sol, LD, and BIC) were loaded differently in the stoop-in-place and stoop-right tasks, perhaps because of some nonlinear interaction between stoop and locomotion. This component had a relatively long duration ( $>20 \%$ of the cycle), consistent with the longer duration of component 6 in the stoop-right task (Fig. $6 C)$. One possibility is that this component might actually represent two (or more) closely spaced activation events that could not be resolved by our methods.

The loading of component 1 in the stoop-in-place task showed a positive correlation with the loading of component 1 in the stoop-right task $(r=0.32$ for 12 muscles that were significantly loaded on the two components, possibly because this component occurs during the stance phase in both behaviors), but the loading was uncorrelated $(r=<0.09)$ with all of the other component loadings in the stoopright task (Fig. 10 A, bottom panels).

For the step-in-place, the right foot was lifted to about the same height as in the obstacle task and then dropped in a continuous movement. The time window was chosen so that it covered all duration of vertical foot motion and the same interval preceding the movement (Fig. $10 \mathrm{~B}, \mathrm{VM}_{\mathrm{y}}$ displacement). EMG data were timeinterpolated over this time window to fit a normalized 200-point time base. In this task, we observed three temporal components (Fig. $10 \mathrm{~B}$ ) that accounted for $>65 \%$ of the variance, with the higher-order components being variable from trial to trial. The timing of component 1 corresponds to a redistribution of the body weight between the two legs before the foot lift, typical also for gait initiation. Component 2 is timed to the foot lift, whereas component 3 may reflect muscle activation needed to terminate the movement at foot contact with a ground. Interestingly, a temporal sequence of the main peaks of these three components resembled that of the normal walk, suggesting that there might be some correspondence of components when the two tasks (locomotion and foot lift) are performed simultaneously (i.e., in the obstacle task).

The peak times of the three in-place components relative to the onset of foot lift were $-17 \%,-3 \%$, and approximately 
Similarities (correlations) of weighting coefficients of 5 basic activation components to those during normal walk
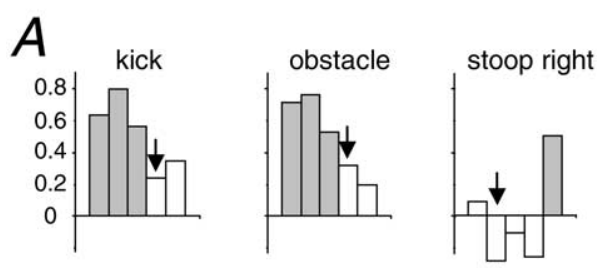

$\square>0.4$
$\square<0.4$

\section{$B$}
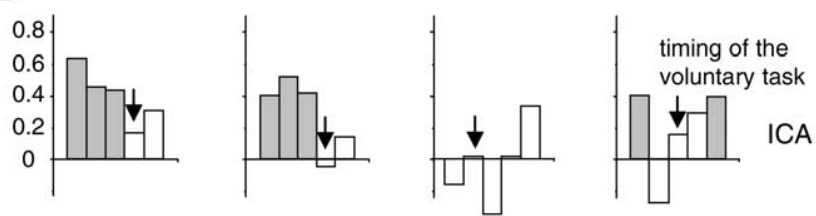

C
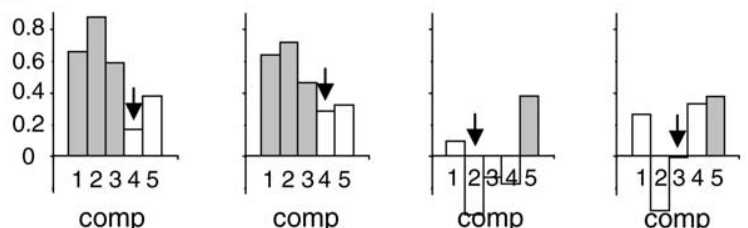

NMF

Figure 9. Similarities (correlations) of weighting coefficients (across 31 muscles) of the five basic temporal components (comp) in all voluntary tasks with those during normal walk. $\boldsymbol{A}-\boldsymbol{C}$ indicate the FA, ICA, and NMF algorithms, respectively. Arrows indicate the activation component closest to the timing of the voluntary task. Relatively high weighting coefficients $(>0.4)$ are shown in gray. Note that, in general, weighting coefficients (loadings) were more severely affected for components neighboring the timing of the voluntary task.

$+55 \%$ of the cycle duration. These correspond to the peak times of components 2,6 , and 1 relative to foot lift in the obstacle task ( $-20,0$, and $+49 \%$, respectively). Thus, component 2 (step-inplace) lines up with the foot lift in the same way as component 6 (obstacle). Moreover, the relative timing of components 1 and 3 (step-in-place) corresponds to the relative timing of components 1 and 2 in the obstacle task. The peak times of these components are also separated by a comparable fraction of the cycle [32\% of the cycle in the obstacle task (Fig. 6C) and 72\% (29\%) in the step-in-place task).

The loadings of components 1 and 3 (step-in-place) were also quite similar to the loadings of components 2 and 1 , respectively, in both the obstacle $(r=0.67$ and 0.51$)$ and normal walk $(r=$ 0.63 and 0.58 ) tasks, whereas component 2 loading was highly correlated only with component 6 loading in the obstacle task $(r=0.7)$ (Fig. $10 B$, bottom). Note that the loadings were highly correlated across subjects (on average, $r=0.94 \pm 0.10$ for components 1 and 2 during obstacle and normal walk), indicating a rather consistent distribution of these components across muscles in different subjects for these tasks.

Thus, temporal components associated with the voluntary task were either synchronous with the timing of the locomotor components or had an additional timing pattern that was not represented in the original locomotor cycle. This is consistent with the idea that neural elements in the central pattern generator (CPG) may be shared with those for a different behavior (Stein and Smith, 1997; d'Avella and Bizzi, 2005).

\section{Discussion}

Our basic finding was that five basic activation timing patterns underlying locomotion were invariantly present when voluntary tasks were performed during locomotion. Moreover, the identification of these activation patterns did not depend on the method used to extract independent components from the EMG data. The voluntary tasks were accompanied by an additional sixth activation component and by different loadings on the five basic temporal components.

\section{Appearance of a new activation component}

The appearance of new task-specific components suggests that the basic locomotion timing pattern was not adequate for a coordinated voluntary task. Nevertheless, the voluntary task did not obliterate or alter the underlying locomotion timing: it simply added to it. This is clear in the FA results (Fig. 6A) and also in many of the EMG records (Fig. 4) showing additional (voluntary) components to the muscle activation patterns of normal walking (e.g., BF, Vmed, and TA).

The task that involved the least perturbation of the locomotion cycle was the kick. Muscle activation at the beginning of swing and just before the kick was heavily weighted in more of the active muscles than during normal walking. In addition to a stronger activation of the muscles normally active in swing (e.g., TA, SART), additional muscles were activated with the same timing (e.g., RF, Vmed, FDB) (Fig. 4). They may therefore represent a component of the voluntary task that is coincident with the locomotion timing. Another part of the task is represented by component 6 , a new activation component not present in locomotion without the kick. It corresponds to the timing of contractions mostly in trunk and shoulder muscles during the kick that are not coincident with the locomotion timing (Fig. 4). Thus, it may reflect a postural compensatory response after the kick.

A new component (component 6) accounted for a much larger fraction of the variance in the other tasks, indicating that the timing of voluntary contractions did not correspond with the locomotion timing. To gain some insight about this, we also examined two of the voluntary tasks separately from forward locomotion, the stoop-in-place and the step-in-place. We found that two components accounted for most of the consistent muscle activation in the stoop-in-place task. The timing of one of them relative to the stoop (Fig. $10 \mathrm{~A}$, comp 2) was similar to the timing of component 6 in the stoop-right task. Moreover, the weightings of this component on the active muscles were also quite similar to those of component 6 (Fig. 10). It suggests that the stoop requires a timing of muscle activation that is different from that of normal walking, the difference being expressed in the combined task by a new activation component with distinct timing. We found a similar correspondence for the step-in-place task, in which components 1 and 3 seemed to be synchronized with the locomotor cycle, whereas component 2 was strictly timed to the onset of foot lift and required a specific muscle activation. This component corresponded then with component 6 in the obstacle task.

This analysis suggests that the recruitment of muscles for the voluntary tasks during locomotion may take two forms. (1) It may coincide with the characteristic timing of the locomotion activation components relative to the step cycle, and thereby alter their muscle loading patterns. This would assure that the voluntary task is appropriately coordinated with the locomotion. (2) The recruitment may occur with a different timing when the locomotion timing is not sufficient for all aspects of the tasks. The finding that the voluntary movements did not alter the timing of the locomotion components implies that the timing was not strongly influenced by the sensory feedback generated by these movements. 
$A$ stoop-in-place

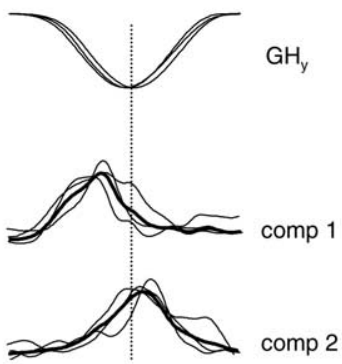

$B$ step-in-place (single foot lift)

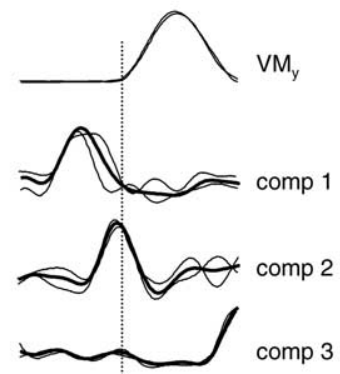

correlation of weighting coefficients across conditions

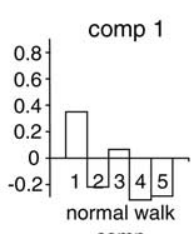

comp
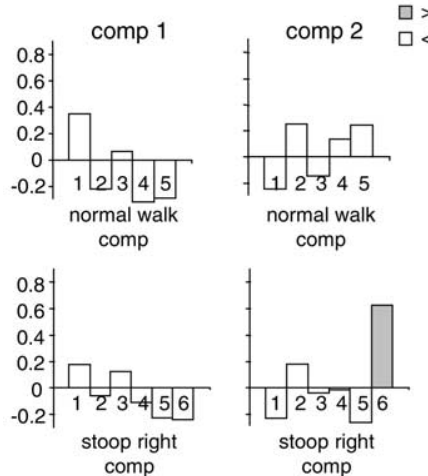

correlation of weighting coefficients across conditions
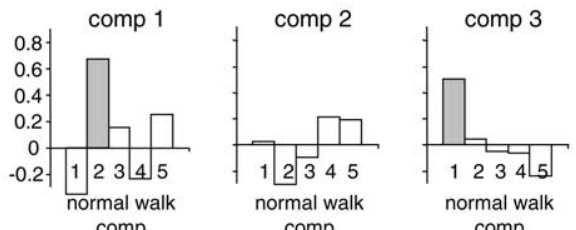

comp
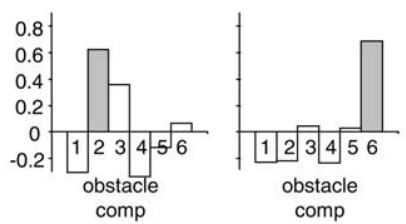

Figure 10. Comparison of reach-and-grasp $(\boldsymbol{A})$ and foot-lift $(\boldsymbol{B})$ movements performed during walking (stoop right and obstacle, respectively) and standing (stoop-in-place and step-in-place). From top to bottom, the superimposed vertical shoulder $\left(\mathrm{GH}_{\mathrm{y}} ; \boldsymbol{A}\right)$ or foot $\left(\mathrm{VM}_{\mathrm{y}} ; \boldsymbol{B}\right)$ displacements, activation components, and correlations between their loadings across muscles with loadings of other components in other tasks are shown. Components 1 and 2 for stoop-in-place accounted for 34 and $30 \%$ of the total variance, respectively. Components $1-3$ for step-in-place accounted for 18,26 , and $22 \%$ of the total variance. Bold lines correspond to the ensemble averaged (across 3 subjects in $\boldsymbol{A}$ and 2 subjects in $\boldsymbol{B}$ ) components. The dotted vertical line corresponds to the timing of the voluntary task (the onset of the vertical object movement in $\boldsymbol{A}$ and of the foot lift in $\boldsymbol{B}$ ). Relatively high weighting coefficients $(>0.4$ ) are marked in gray and might underline a similar group of muscles (muscle synergies) involved in these activation patterns. comp, Component.

\section{Proposed superposition of motor programs}

The analysis of component timing and coordination suggests a control strategy that generates voluntary tasks during locomotion by superimposing a voluntary activation program onto the basic locomotion activation. According to this interpretation, the five locomotion components represent the elements of a motor program for locomotion consisting of a particular activation sequence that is distributed to various muscles depending on the biomechanical demands of the locomotion in progress (Fig. 11 A) (Ivanenko et al., 2004). This sequence is likely to be partly determined in some way by proprioceptive feedback (Pearson, 1995; Duysens and Van de Crommert, 1998), but the results emphasize a dominant timing sequence that is basically unperturbed by major differences in sensory input or postural demands.

Thus, it appears that spinal mechanisms may somehow compensate for or anticipate both sensory and descending signals so they conform to a specific timing pattern. Although our results do not point to specific underlying mechanisms, recent experimental evidence from the cat suggests some possibilities. The finding of critical periods for the effectiveness of sensory input in modifying CPG activity during fictive locomotion (Saltiel and Rossignol, 2004) implies that inputs to the CPG circuitry are somehow shaped to conform to anticipated biomechanical events. Drew et al. (2004) have also implicated both brainstem and spinal CPGrelated networks in the coordination of voluntary and posture-related activities during locomotion. They suggest that the cyclic excitability of spinal interneurons during locomotion may determine the phase during which descending signals are effective, thereby shaping them to conform to the locomotor activation timing. Descending motor commands may also be coordinated with locomotion activation through the influence of ascending pathways and the corresponding rhythmical modulation of the central structures during locomotion (Orlovsky et al., 1999; Dietz, 2002).

The spinal networks responsible for the locomotion timing pattern are likely to be distributed across several spinal segments linked by long propriospinal connections [as suggested by Nathan et al. (1996)] including both descending and sensory inputs (Kiehn et al., 1998; Orlovsky et al., 1999; Dietz, 2002). Some evidence for this may be seen in spinal cord injury patients with both complete and incomplete spinal transections (Ivanenko et al., 2003). The same five temporal components account for the timing of muscle activation despite a vast modification of individual motor patterns in these patients (Grasso et al., 2004). Moreover, muscles both rostral and caudal to the lesions could be strongly weighted on a given component.

The sequence of activation components in locomotion bears some resemblance to the sequential activation of motor primitives proposed to account for a variety of movements in decerebrate and spinal frogs (Hart and Giszter, 2004). The frog components consist of short duration, synchronous activations of muscle synergies. However, unlike the locomotion activation, the activation was found to have a single characteristic time course for all tasks. Instead, we found that activation timing and duration are normalized to the duration of the gait cycle, consistent with the scaling of muscle activation waveforms with speed (Wallen and Williams, 1984; Orlovsky et al., 1999; Ivanenko et al., 2004). We also found that the grouping of muscles activated by each component was task dependent and may have represented muscle synergies; however, a more direct interpretation of our results is that locomotion pattern generators activate motoneurons via a distribution network that sets weightings according to feedback and feedforward parameters (Fig. 11A) (Perret and Cabelguen, 1980; Patla et al., 1985; Hultborn, 2001; Poppele and Bosco, 2003; Ivanenko et al., 2004).

Our proposal is that separate motor programs having distinct activation commands and timing may be superimposed to generate combined, coordinated movements (Fig. $11 B, C$ ). Thus, the combined locomotion and voluntary tasks may be controlled by a combination of the underlying motor programs for both, each with a characteristic activation timing (Fig. 11C). When the timing is basically the same for the components of a combined task, 
the result will have the same activation timing (Fig. 11C, bottom, light gray components), but, in general, the muscles activated will be different, and perhaps their sources of activation will also be different. Any discrepancy between activation timing in the component tasks results in additional activation components (Fig. 11C, dark gray components). Coordinating the components of the combined task is assured by synchronizing the activation timings. We believe that the results presented here are consistent with this basic idea.

There are, however, many reasons to expect that these compound tasks should not relate directly to a simple sum of the component tasks. For one, the proprioceptive feedback for locomotion is not the same when the locomotion includes a stoop or higher step. Moreover, the biomechanical demands of the modified locomotion kinematics are basically different from those of normal walking. In fact, whereas the stoop-in-place produced components that loaded similarly to the first and sixth components for stoop right, the correspondence was not exact. The difference may reflect differences in the effects of feedback on the weightings, or it may reflect a nonlinear interaction between stoop-grasp and locomotion. However, we showed in a previous study that the different biomechanical demands of locomotion speed and body weight support also did not alter the basic locomotion program. In those cases, too, the demands were met through the selection of muscles to be activated at the times dictated by the five basic components. What is suggested by these results is that the selection of muscle synergies is downstream from the processes that generate activation timings.

\section{References}

Basmajian JV, De Luca CJ (1985) Muscles alive: their functions revealed by electromyography, Ed 5. Baltimore: Williams and Wilkins.

Bell AJ, Sejnowski TJ (1995) An information-maximization approach to blind separation and blind deconvolution. Neural Comput 7:1129-1159.

Bernstein N (1967) The co-ordination and regulation of movements. Oxford: Pergamon.

Bianchi L, Angelini D, Lacquaniti F (1998) Individual characteristics of human walking mechanics. Pflügers Arch 436:343-356.

Bizzi E, Tresch MC, Saltiel P, d'Avella A (2000) New perspectives on spinal motor systems. Nat Rev Neurosci 1:101-108.

Borghese NA, Bianchi L, Lacquaniti F (1996) Kinematic determinants of human locomotion. J Physiol (Lond) 494:863-879.

Bresler E, Frankel JB (1950) The forces and moments in the leg during level walking. J Appl Mech 72:27-36.

Chau T (2001) A review of analytical techniques for gait data. Part 1: Fuzzy, statistical and fractal methods. Gait Posture 13:49-66.

Chou LS, Draganich LF (1997) Stepping over an obstacle increases the motions and moments of the joints of the trailing limb in young adults. J Biomech 30:331-337.

d'Avella A, Bizzi E (2005) Shared and specific muscle synergies in natural motor behaviors. Proc Natl Acad Sci USA 102:3076-3081.

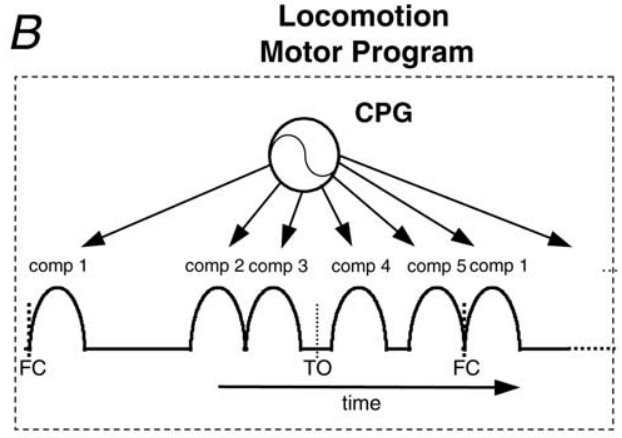

Voluntary Motor Programs

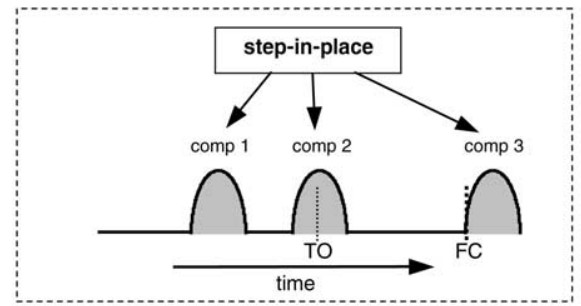

Combined Programs

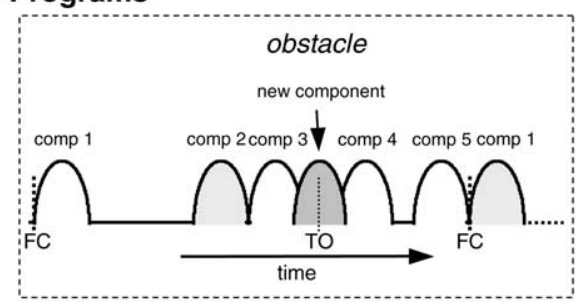

11. Muscle activation. $\boldsymbol{A}$. Activation components. The activation pulse acts via a distribution network to activate a 列 (light gray) or different (gray or white). comp, Component; FC, foot contact; T0, toe off.

d'Avella A, Saltiel P, Bizzi E (2003) Combinations of muscle synergies in the construction of a natural motor behavior. Nat Neurosci 6:300-308.

Davis BL, Vaughan CL (1993) Phasic behavior of EMG signals during gait: use of multivariate statistics. J EMG Kinesiol 3:51-60.

Delorme A, Makeig S (2004) EEGLAB: an open source toolbox for analysis of single-trial EEG dynamics including independent component analysis. J Neurosci Methods 134:9-21.

Dietz V (2002) Do human bipeds use quadrupedal coordination? Trends Neurosci 25:462-467.

Drew T, Prentice S, Schepens B (2004) Cortical and brainstem control of locomotion. Prog Brain Res 143:251-261.

Duysens J, Van de Crommert HW (1998) Neural control of locomotion: the central pattern generator from cats to humans. Gait Posture 7:131-141.

Giszter SF, Moxon KA, Rybak IA, Chapin JK (2001) Neurobiological and neurorobotic approaches to control architectures for a humanoid motor system. Robotics Auton Syst 37:219-235.

Grasso R, Ivanenko YP, Zago M, Molinari M, Scivoletto G, Castellano V, Macellari V, Lacquaniti F (2004) Distributed plasticity of locomotor pattern generators in spinal cord injured patients. Brain 127:1019-1034.

Hart CB, Giszter SF (2004) Modular premotor drives and unit bursts as primitives for frog motor behaviors. J Neurosci 24:5269-5282.

Hultborn H (2001) State-dependent modulation of sensory feedback. J Physiol (Lond) 533:5-13.

Ivanenko YP, Grasso R, Zago M, Molinari M, Scivoletto G, Castellano V, Macellari V, Lacquaniti F (2003) Temporal components of the motor 
patterns expressed by the human spinal cord reflect foot kinematics. J Neurophysiol 90:3555-3565.

Ivanenko YP, Poppele RE, Lacquaniti F (2004) Five basic muscle activation patterns account for muscle activity during human locomotion. J Physiol (Lond) 556:267-282.

Kaiser HF (1974) Analysis of factorial simplicity. Psychometrika 39:31-36.

Kargo WJ, Giszter SF (2000) Rapid correction of aimed movements by summation of force-field primitives. J Neurosci 20:409-426.

Kendall FP, McCreary EK, Provance PG (1993) Muscles. Testing and function. Baltimore: Williams and Wilkins.

Kiehn O, Harris-Warrick RM, Jordan LM, Hultborn H, Kudo N, editors (1998) Neuronal mechanisms for generating locomotor activity. Ann NY Acad Sci 860:1-573.

Krishnamoorthy V, Latash ML, Scholz JP, Zatsiorsky VM (2003) Muscle synergies during shifts of the center of pressure by standing persons. Exp Brain Res 152:281-292.

Lacquaniti F, Grasso R, Zago M (1999) Motor patterns in walking. News Physiol Sci 14:168-174.

Lacquaniti F, Ivanenko YP, Zago M (2002) Kinematic control of walking. Arch Ital Biol 140:263-272.

Latash ML (1999) On the evolution of the notion of synergy. In: Motor control, today and tomorrow (Gantchev GN, Mori S, Massion J, eds), pp 181-196. Sofia, Bulgaria: Academic Publishing House "Prof. M. Drinov."

Lee DD, Seung HS (1999) Learning the parts of objects by non-negative matrix factorization. Nature 401:788-791.

Lemay MA, Grill WM (2004) Modularity of motor output evoked by intraspinal microstimulation in cats. J Neurophysiol 91:502-514.

Makeig S, Debener S, Onton J, Delorme A (2004) Mining event-related brain dynamics. Trends Cogn Sci 8:204-210.

McFadyen BJ, Carnahan H (1997) Anticipatory locomotor adjustments for accommodating versus avoiding level changes in humans. Exp Brain Res 114:500-506.

McFadyen BJ, Winter DA (1991) Anticipatory locomotor adjustments during obstructed human walking. Neurosci Res Commun 9:37-44.

Merkle LA, Layne CS, Bloomberg JJ, Zhang JJ (1998) Using factor analysis to identify neuromuscular synergies during treadmill walking. J Neurosci Methods 82:207-214.

Nathan PW, Smith M, Deacon P (1996) Vestibulospinal, reticulospinal and descending propriospinal nerve fibres in man. Brain 119:1809-1833.

Olree KS, Vaughan CL (1995) Fundamental patterns of bilateral muscle activity in human locomotion. Biol Cybern 73:409-414.

Orlovsky GN, Deliagina TG, Grillner S (1999) Neural control of locomotion. From mollusc to man. Oxford: Oxford UP.

Patla AE (1985) Some characteristics of EMG patterns during locomotion: implications for the locomotor control process. J Mot Behav 17:443-461.

Patla AE, Calvert TW, Stein RB (1985) Model of a pattern generator for locomotion in mammals. Am J Physiol 248:R484-R494.

Patla AE, Prentice SD, Robinson C, Neufeld J (1991) Visual control of loco- motion: strategies for changing direction and for going over obstacles. J Exp Psychol Hum Percept Perform 17:603-634.

Pearson KG (1995) Proprioceptive regulation of locomotion. Curr Opin Neurobiol 5:786-791.

Perret C, Cabelguen JM (1980) Main characteristics of the hindlimb locomotor cycle in the decorticate cat with special reference to bifunctional muscles. Brain Res 187:333-352.

Poppele R, Bosco G (2003) Sophisticated spinal contributions to motor control. Trends Neurosci 26:269-276.

Prochazka A, Gritsenko V, Yakovenko S (2002) Sensory control of locomotion: reflexes versus higher-level control. Adv Exp Med Biol 508:357-367.

Saltiel P, Rossignol S (2004) Critical points in the forelimb fictive locomotor cycle and motor coordination: evidence from the effects of tonic proprioceptive perturbations in the cat. J Neurophysiol 92:1329-1341.

Shiavi R, Griffin P (1981) Representing and clustering electromyographic gait patterns with multivariate techniques. Med Biol Eng Comput 19:605-611.

Stein PS, Smith JL (1997) Neural and biomechanical control strategies for different forms of vertebrate hindlimb motor tasks. In: Neurons, networks, and motor behavior (Stein PSG, Grillner S, Selverston AI, Stuart DG, eds), pp 61-73. Cambridge, MA: MIT.

Ting LH, Macpherson JM (2005) A limited set of synergies for force control during a postural task. J Neurophysiol 93:609-613.

Tresch MC, Saltiel P, d'Avella A, Bizzi E (2002) Coordination and localization in spinal motor systems. Brain Res Brain Res Rev 40:66-79.

van Hedel HJ, Biedermann M, Erni T, Dietz V (2002) Obstacle avoidance during human walking: transfer of motor skill from one leg to the other. J Physiol (Lond) 543:709-717.

Wallen P, Williams TH (1984) Fictive locomotion in the lamprey spinal cord in vitro compared with swimming in the intact spinal animal. J Physiol (Lond) 347:225-239.

Winter DA (1991) The biomechanics and motor control of human gait: normal, elderly and pathological. Waterloo, Ontario, Canada: Waterloo Biomechanics.

Winter DA, Yack HJ (1987) EMG profiles during normal human walking: stride-to-stride and inter-subject variability. Electroencephalogr Clin Neurophysiol 67:402-411.

Wootten ME, Kadaba MP, Cochran GV (1990) Dynamic electromyography. II. Normal patterns during gait. J Orthop Res 8:259-265.

Yakovenko S, Mushahwar V, VanderHorst V, Holstege G, Prochazka A (2002) Spatiotemporal activation of lumbosacral motoneurons in the locomotor step cycle. J Neurophysiol 87:1542-1553.

Zatsiorsky V, Seluyanov V, Chugunova L (1990) In vivo body segment inertial parameters determination using a gamma-scanner method. In: Biomechanics of human movement: applications in rehabilitation, sports and ergonomics (Berme N, Cappozzo A, eds), pp 186-202. Worthington, OH: Bertec.

Zehr EP, Duysens J (2004) Regulation of arm and leg movement during human locomotion. The Neuroscientist 10:347-361. 